\title{
PROBING T TAURI ACCRETION AND OUTFLOW WITH 1 MICRON SPECTROSCOPY \\ Suzan Edwards, ${ }^{1,2,3}$ William Fischer, ${ }^{4}$ Lynne Hillenbrand, ${ }^{2,5}$ and John Kwan ${ }^{4}$ \\ Received 2006 January 27; accepted 2006 March 29
}

\begin{abstract}
In a high-dispersion $1 \mu \mathrm{m}$ survey of 39 classical T Tauri stars (CTTSs) veiling is detected in $80 \%$ of the stars, and $\mathrm{He}_{\mathrm{I}} \lambda 10830$ and $\mathrm{P} \gamma$ line emission in $97 \%$. On average, the $1 \mu \mathrm{m}$ veiling exceeds the level expected from previously identified sources of excess emission, suggesting the presence of an additional contributor to accretion luminosity in the star-disk interface region. Strengths of both lines correlate with veiling, and at $\mathrm{P} \gamma$ there is a systematic progression in profile morphology with veiling. He $\mathrm{I} \lambda 10830$ has an unprecedented sensitivity to inner winds, showing blueshifted absorption below the continuum in $71 \%$ of the CTTSs, compared to $0 \%$ at $\mathrm{P} \gamma$. This line is also sensitive to magnetospheric accretion flows, with redshifted absorption below the continuum found in $47 \%$ of the CTTSs, compared to $24 \%$ at $\mathrm{P} \gamma$. The blueshifted absorption at $\mathrm{He}$ I $\lambda 10830$ shows considerable diversity in its breadth and penetration depth into the continuum, indicating that a range of inner wind conditions exist in accreting stars. We interpret the broadest and deepest blue absorptions as formed from scattering of the $1 \mu \mathrm{m}$ continuum by outflowing gas whose full acceleration region envelopes the star, suggesting radial outflow from the star. In contrast, narrow blue absorption with a range of radial velocities more likely arises via scattering of the $1 \mu \mathrm{m}$ continuum by a wind emerging from the inner disk. Both stellar and disk winds are accretion powered, since neither is seen in nonaccreting WTTSs and among the CTTSs helium strength correlates with veiling.
\end{abstract}

Subject headings: planetary systems: protoplanetary disks — stars: formation — stars: pre-main-sequence — stars: winds, outflows

\section{INTRODUCTION}

In the formation of a star and its attendant planetary system the phase of disk accretion is always accompanied by mass outflow. Outflow collimation perpendicular to the disk plane and correlations between disk accretion rate and wind mass-loss rate, with a rough proportion of $\dot{M}_{w} / \dot{M}_{\text {acc }} \sim 0.1$, certify the intimate relation between these two phenomena (Richer et al. 2000; Cabrit et al. 1990). Although the coupling of accretion and outflow is well documented, the means of launching the outflow remains unknown and is one of the major unsolved mysteries of star formation. There is a strong motivation to understand where and how energetic winds originate in accretion disk systems, since they are strong contenders for a major role in mass and angular momentum transport in the disk (Ferreira \& Casse 2004), for stellar spindown (Königl 1991; Shu et al. 1994; Matt \& Pudritz 2005), for heating the disk atmosphere (Glassgold et al. 2004), for hastening disk dissipation (Hollenbach et al. 2000), and for disrupting infalling material from the collapsing molecular cloud core (Terebey et al. 1984).

Most outflow models tap magnetocentrifugal ejection as the heart of the launching mechanism, where rotating fields in the disk fling material along inclined field lines and transport angular momentum away from the disk. A distinguishing characteristic among disk wind models is the location in the system where mass loading onto field lines occurs. This takes place either from the inner disk over a range of radii (Königl \& Pudritz 2000) or

\footnotetext{
${ }^{1}$ Five College Astronomy, Smith College, Northampton, MA 10163; sedwards@smith.edu.

2 Visiting Astronomer, Keck Observatory.

3 Guest Observer, Gemini/Keck-NIRSPEC program.

4 Five College Astronomy, University of Massachusetts, Amherst, MA, 01003; kwan@astro.umass.edu,wfischer@astro.umass.edu.

5 Department of Astronomy, California Institute of Technology, Pasadena, CA 91125; lah@astro.caltech.edu.
}

from the radius at which the stellar magnetosphere truncates the disk, lifting most of the accreting material toward the star into magnetic funnel flows and ejecting the rest along opened stellar field lines emerging from this point (Shu et al. 1994). Others have explored the possibility that accretion-driven winds emerge via magnetohydrodynamic acceleration from the star rather than or in addition to the disk (Hirose et al. 1997; Kwan \& Tademaru 1988; Matt \& Pudritz 2005; Romanova et al. 2005; von Rekowski \& Brandenburg 2006). Establishing the launch region for accretion powered winds is thus of considerable interest, since this can discriminate among the various wind theories and elucidate how angular momentum is removed from the star-disk environment.

Knowledge of accretion driven winds comes primarily from spatially extended collimated atomic and molecular jets comprised of high-velocity $\left(200-400 \mathrm{~km} \mathrm{~s}^{-1}\right)$ outflowing material undergoing internal shocks (Hartigan et al. 1995, 2004; Reipurth $\&$ Bally 2001). These tracers yield basic wind energetics and establish the fundamental connection between $\dot{M}_{w}$ and $\dot{M}_{\text {acc }}$ but provide only indirect information on the wind-launching region. An intriguing result comes from spectroscopy at high spatial resolution of T Tauri "microjets" revealing velocity asymmetries that have been interpreted as coherent rotation of the jet as would be imparted from an MHD disk wind launched from moderate disk radii of 0.5-2 AU (Coffey et al. 2004). However, recent three-dimensional simulations of line profiles formed in precessing jets with variable ejection velocities demonstrate that these conditions can produce similar velocity gradients, thereby calling into question the implied support for magnetocentrifugal wind acceleration models (Cerqueira et al. 2006).

Direct probes of the launch region can, however, only be made via high-resolution spectroscopy. Confoundingly, most spectral features from sub-astronomical unit regions have kinematic signatures deriving from rotation or accretion rather than outflow. Near-infrared lines of $\mathrm{CO}$ and $\mathrm{H}_{2} \mathrm{O}$ are modeled as arising from inner accretion disks in Keplerian rotation at radii from 0.1 to 
2 AU, precisely the region where the rotating jets are predicted to arise (Najita et al. 2003; Carr et al. 2004). Although modeling these features requires turbulent broadening and warm disk chromospheres, their symmetry reveals no evidence for outflowing gas from the upper disk layers. A possible signature of a wind emerging from the disk over radii ranging to tens of astronomical units, is seen in the "low-velocity component" (LVC) of CTTS forbidden lines, distinguished from the "high-velocity component" (HVC) by smaller blueshifts $\left(\sim 10 \mathrm{~km} \mathrm{~s}^{-1}\right)$, smaller spatial extensions, and line ratios indicative of formation under conditions of higher density, although its interpretation as the launch of an extended disk wind remains unconfirmed (Hartigan et al. 1995; Kwan \& Tademaru 1995).

However, outflow velocities of $200-500 \mathrm{~km} \mathrm{~s}^{-1}$ characteristic of jets and HVC forbidden line emission point to an origin deeper in the potential well of the accretion disk system - either the very inner disk, the star-disk interface, or the star itself. These regions, where the stellar magnetosphere controls the accretion flow onto the star, are probed via permitted atomic emission lines from the infrared through to the ultraviolet and include a wide range of ionization states. Their line profiles, typically with strong and broad emission sometimes accompanied by redshifted absorption below the continuum and/or narrow centered emission, are attributed to formation in magnetospheric funnel flows and accretion shocks on the stellar surface (Edwards et al. 1994; Muzerolle et al. 2001a; Herczeg et al. 2002, 2005). Until recently the only clear evidence for inner winds was blueshifted absorption superposed on the broad emission lines of $\mathrm{H} \alpha, \mathrm{Na} \mathrm{D}$, $\mathrm{Ca}$ II $\mathrm{H}$ and $\mathrm{K}$, and $\mathrm{Mg}$ II $h$ and $k$, signifying the presence of a highvelocity wind close to the star (Calvet 1997; Ardila et al. 2002) but yielding little information on the nature or location of the windlaunching region.

Somewhat surprisingly, robust diagnostics of the inner wind in accretion disk systems are turning out to be lines formed under conditions of high excitation-He I and possibly also $\mathrm{C}$ III and O vi (Edwards et al. 2003; Dupree et al. 2005) - arising in regions where temperatures exceed $15,000 \mathrm{~K}$. The first indication of inner winds from regions with high excitation came from a study of $\mathrm{He}_{\mathrm{I}} \lambda \lambda 5876$ and 6678 in a sample of 31 accreting CTTSs. (Beristain et al. 2001, hereafter BEK01). The high excitation of the helium lines restricts line formation to a region either of high temperature or close proximity to a source of ionizing radiation. In spite of these restrictions, the helium emission lines were found to have a composite origin, including contributions from a wind, from the funnel flow, and from an accretion shock.

Confirmation that broad helium emission traces inner winds in accreting stars came from observations of another line in the $\mathrm{He} \mathrm{I}$ triplet series, $\lambda 10830$, which is a far more sensitive diagnostic of outflowing gas. This line, immediately following $\lambda 5876$ in a recombination-cascade sequence, has a highly metastable lower level, setting up a favorable situation for tracing outflowing gas in absorption. An initial look at six high accretion rate stars (AS 353A, DG Tau, DL Tau, DR Tau, HL Tau, and SVS 13) focused on examples of $\mathrm{He}$ I $\lambda 10830$ profiles with exceptionally strong P Cygni wind signatures (Edwards et al. 2003). More recently, He I $\lambda 10830$ profiles for two additional CTTSs (TW Hya and T Tau) were also shown to have P Cygni profiles (Dupree et al. 2005). Both of these studies concluded that the winds tracked by helium absorption likely arise as a wind expanding radially away from the star, tracing the wind through its full acceleration from rest to several hundred kilometers per second.

In this manuscript we present results of a census of $\mathrm{He}$ I $\lambda 10830$ in 39 CTTSs and 6 WTTSs plus 3 Class I sources from spectra collected with Keck II's NIRSPEC. We find that this line is exceptionally sensitive not only to winds but also to infalling gas. Our spectra also yield simultaneous $\mathrm{P} \gamma$ profiles and $1 \mu \mathrm{m}$ continuum excess (veiling), providing a good combination of features to probe the relation between inner winds and accretion in young low-mass stars. The remainder of the paper is organized as follows: In $\S 2$ we discuss observations and data reduction, including sample selection and a description of the determination of the excess continuum emission and residual emission profiles; in $\S 3$ we give the accretion diagnostics: $\mathrm{P} \gamma$ and $1 \mu \mathrm{m}$ veiling; in $\S 4$ the wind diagnostic: He $\mathrm{I} \lambda 10830$; in $\S 5$ the Class I sources; in $\S 6$ we discuss magnetospheric accretion contributions to $\mathrm{P} \gamma$ and $\mathrm{He}_{\mathrm{I}} \lambda 10830$ and wind contributions to He I $\lambda 10830$; in $\S 7$ the origin of $1 \mu \mathrm{m}$ veiling; and in $\S 8$ we offer our conclusions.

\section{OBSERVATIONS AND DATA REDUCTION}

Near-infrared echelle spectra of 39 classical T Tauri stars (CTTSs), 6 weak T Tauri stars (WTTSs), and 3 Class I sources were acquired with NIRSPEC on Keck II (McLean et al. 1998), using the $\mathrm{N} 1$ filter ( $Y$ band) with wavelength coverage from 0.95 to $1.12 \mu \mathrm{m}$ at $R=25,000\left(\Delta V=12 \mathrm{~km} \mathrm{~s}^{-1}\right)$. The detector is a $1024 \times 1024$ pixel InSb chip, and the spectrograph slit was $0.43^{\prime \prime} \times 12^{\prime \prime}$ Spectra were taken in nodded pairs in an ABBA pattern, with the target separated by $5^{\prime \prime}$ in each AB pair. Typical exposure times were $300 \mathrm{~s}$, integrated in each case to acquire $\mathrm{S} / \mathrm{N} \sim 70-80$ in the reduced one-dimensional (1D) spectra. All objects were observed on a three-night run in 2002 November by Hillenbrand \& Edwards. Three of our program stars were also observed on 2001 November 22 for Edwards and A. Dupree by the Gemini-Keck queue observers T. Geballe and M. Takamiya (see Edwards et al. 2003). In 2002 spectra were acquired without the image derotator, due to equipment malfunction. Although this prevents study of spatially extended emission, as reported for DG Tau (Takami et al. 2002), it is not a problem for extraction of a spectrum from the central point source, where most of the line emission and all of the line absorption arises.

Spectra were processed in IRAF using a modified version of a script originally provided by M. Takamiya. Data reduction steps included spatial rectification of the two-dimensional (2D) images, wavelength calibration with a $\mathrm{Xe} / \mathrm{Ar} / \mathrm{Kr} / \mathrm{Ne}$ arc lamp spectrum, correction for telluric emission, telluric absorption, and instrumental fringing, and extraction to 1D spectra using IRAF's apall package. Telluric emission was removed by subtraction of each $\mathrm{AB}$ pair of images, yielding two sky-subtracted spectra that were then co-added after spatial rectification and wavelength calibration. Telluric absorption features were removed by dividing the rectified, extracted, co-added, wavelength-calibrated spectra by the spectrum of an early-type standard observed at a similar air mass.

Division by a telluric standard also corrected the sinusoidal fringing pattern characterized by a spacing of $\sim 1.5 \AA$ (10 pixels) and an amplitude $\sim 3 \%$ of the continuum) for the 2002 data, where there was a good alignment $(\leq 0.7$ pixels) of the fringing pattern between the target and telluric standard. For the few 2001 spectra taken under queue observing, the fringe pattern was misaligned between target and standard by 1.5 to 4.5 pixels $(0.5$ to 1.5 times the instrumental resolution). For these spectra we determined the amount of fringe shift in wavelength by cross-correlating the target and standard spectra in a region free of spectral features, and found that the telluric absorption features shared the same misalignment. Consequently, after shifting the standard by the requisite amount, division by the standard conveniently removed both contaminating features. A final problem occasionally arose for stars observed at high air mass, when weak residual telluric 
features in the vicinity of the $\mathrm{He}_{\mathrm{I}} \lambda 10830$ and $\mathrm{P} \gamma$ lines appeared in the reduced spectrum. These were readily removed from the residual profile by determining their strength relative to stronger telluric features in the rest of the spectral order.

Our telluric standards were of early spectral type (O7 to B9), providing a nearly featureless continua over most of the orders yielding a good definition of telluric features. However, they have strong photospheric lines of $\mathrm{He} \mathrm{I} \lambda 10830$ and $\mathrm{P} \gamma$, our two primary spectral diagnostics. We thus took special care to define and remove these broad photospheric features in the standards so that spurious emission would not be induced in our T Tauri spectra. This was accomplished by first creating a synthetic spectrum of each standard where the observed profiles were modeled with a combination of Gaussian and Lorentzian functions, and then dividing the synthetic spectrum into the actual standard spectrum. We judged the fit effective when the final continuumnormalized spectra were flat in these spectral regions to within $1 \%$. An additional correction for helium emission had to be made for HR 130 using a similar technique. The absence of any broad helium or hydrogen emission in our WTTS spectra (discussed in $\S 2.1)$ verifies that this procedure was effective. After removal of all of the above instrumental effects, the definition of the final spectrum is limited only by the signal-to-noise ratio of the target and standard.

Although 13 echelle orders appear on the chip with the N1 filter, the first and last are severely contaminated by telluric absorption. Of the remaining 11 orders, the number and location of arc lamp lines is such that reliable wavelength solutions can be obtained for four full orders and five partial ones. The order extending from 1.081 to $1.096 \mu \mathrm{m}$ contains both our prime spectroscopic diagnostics of $\mathrm{He}$ I $\lambda 10830$ and $\mathrm{P} \gamma$ and also many photospheric features in late-type stars. In addition to this order we identified two additional orders with abundant photospheric lines that we used for redundant estimates of the continuum veiling and determination of the stellar velocity. One, immediately to the blue of our prime order, extends from 1.066 to $1.081 \mu \mathrm{m}$ and has numerous photospheric features and minimal contamination from telluric absorption. A second, although its full extension is from 0.971 to $0.986 \mu \mathrm{m}$, only had sufficient arc line coverage for wavelength calibration from 0.971 to $0.979 \mu \mathrm{m}$. Even with only half the order usable we found it to be preferable to other orders because its strong photospheric features were still detectable when photospheric lines in the other orders were obscured by broad metallic emission features that were sometimes found in stars with the highest continuum veiling.

\subsection{Determination of Excess Continuum Emission and Residual Profiles}

The 39 CTTSs and 6 WTTSs in our sample are identified in Table 1. All but two have spectral types from K0 to M5, with K7/M0 stars comprising the majority (27 stars). The remaining 2 are of solar type, SU Aur (G2) and GW Ori (G5). Most (30) are in the Tau-Auriga star formation complex; the rest are well studied $\mathrm{T}$ Tauri stars from associations including those in Orion and TW Hydra. The sample covers the full range of continuum emission excesses in the optical and ultraviolet found for CTTSs and was selected to overlap extensively with an earlier optical echelle survey of 31 CTTSs appearing in Hartigan et al. 1995 (hereafter HEG95) and BEK01, thereby allowing a comparison of optical and infrared outflow and accretion diagnostics (including profiles of [O I ] $\lambda 6300, \mathrm{H} \alpha$, and $\mathrm{He}$ I $\lambda 5876$ ) for stars common to both studies. The current sample thus includes 30 of the 31 CTTSs in HEG95 and BEK01, identified in Table 1 by an entry for veiling at $5700 \AA\left(r_{V}\right)$. Also included in Table 1 are disk accretion rates determined from veiling, spanning $\dot{M}_{\text {acc }} \sim 10^{-6}$ to $10^{-9} M_{\odot} \mathrm{yr}^{-1}$, mass outflow rates from forbidden lines, spanning $\dot{M}_{w} \sim 10^{-7}$ to $10^{-10} M_{\odot} \mathrm{yr}^{-1}$ and the corresponding ratio of $\dot{M}_{w} / \dot{M}_{\mathrm{acc}}$.

We have determined both the $1 \mu \mathrm{m}$ veiling $r_{Y}$, defined as the ratio of excess to photospheric flux in the $Y$ band, and residual emission profiles at $\mathrm{He}$ I $\lambda 10830$ and $\mathrm{P} \gamma$ for each of our objects. Our method for veiling determination follows Hartigan et al. (1989), matching an echelle order of an accreting star to that of a nonaccreting standard of comparable spectral type to which has been added a constant continuum excess, i.e., the veiling, that reproduces the depth of the photospheric features in the accreting star. We used an interactive IDL routine kindly provided by Russel White to accomplish the requisite velocity shift of the standard via cross-correlation and the requisite rotational broadening of the standard before determining the required level of continuum excess. When a good match between the accreting star and the veiled standard is found, subtraction of the template yields a zerointensity residual spectrum for the accreting star, except in the vicinity of lines arising from accretion-related activity. Integrated equivalent widths for spectral lines were determined with an IDL routine kindly provided by Catrina Hamilton.

In Figure 1 continuum normalized spectra for each of the six WTTSs (LkCa 4, TWA 3, TWA 14, V819 Tau, V826 Tau, and V827 Tau) are shown along with the CTTS GM Aur. Spectra in $\pm 500 \mathrm{~km} \mathrm{~s}^{-1}$ windows are centered on the wavelength of the $\mathrm{He}_{\mathrm{I}} \lambda 10830$ and $\mathrm{P} \gamma$ lines, at rest with respect to the star as determined from the photospheric lines. In each region several photospheric features are marked with vertical lines in the upper spectrum of Figure 1 (GM Aur). In the He I $\lambda 10830$ region the three strongest photospheric features are $\mathrm{Si}$ I $\lambda 10830.1, \mathrm{Na}$ I $\lambda 10837.8$, and $\mathrm{Si}$ I $\lambda$ 10846.8. In the $\mathrm{P} \gamma$ region the three marked lines correspond to $\mathrm{H}_{\mathrm{I}} \lambda 10941.1$ ( $\left.\mathrm{P} \gamma\right), \mathrm{Fe}_{\mathrm{I}} \lambda 10949.8$, and $\mathrm{Mg}$ I $\lambda 10956.3$. The remaining numerous absorption features in that region are part of a broad $\mathrm{CN} 0-0$ band, not noise. V826 Tau is a spectroscopic binary (Mundt et al. 1983), and in our data the photospheric lines are double with a velocity separation of $21 \mathrm{~km} \mathrm{~s}^{-1}$.

None of the six WTTSs or GM Aur have any excess continuum emission $\left(r_{Y}<0.05\right)$, and only two of them have weak emission at $\mathrm{He}_{\mathrm{I}} \lambda 10830$ or $\mathrm{P} \gamma$ above an upper limit of $W_{\lambda}<0.2 \AA$. Both lines are weakly emitting in V826 Tau, with $W_{\lambda}=0.8 \AA$ for helium and $W_{\lambda} \leq 0.3 \AA$ for $\mathrm{P} \gamma$. TWA 14 has only helium in emission, with $W_{\lambda}=0.6 \AA$. The emission equivalent widths for these two stars are several times weaker than the weakest emission found for the CTTSs. Optical He I 25876 emission was also found to be rare among WTTSs, seen in only 3 out of 10 WTTSs by BEK01, including V826 Tau. Both the optical and infrared helium emission may arise in an active chromosphere or a very weak accretion flow.

In earlier studies GM Aur has shown the hallmarks of an accreting CTTS - optical veiling, $\mathrm{O}$ г emission, and strong $\mathrm{H} \alpha$ (HEG95; Gullbring et al. 1998). However, in our NIRSPEC data this is the only one of the 39 CTTSs that shows no line emission in $\mathrm{He}_{\mathrm{I}} \lambda 10830$ or $\mathrm{P} \gamma\left(W_{\lambda}<0.2 \AA\right)$, which we interpret as a sign that accretion has temporarily ceased. Its weak $\mathrm{P} \gamma$ absorption feature is similar in strength to that from the photosphere of an early K star (Hinkle et al. 1995). The spectral energy distribution for GM Aur has been interpreted as indicative of a large (several AU) inner gap in its disk (Rice et al. 2003), so the absence of CTTS characteristics at $1 \mu \mathrm{m}$ in 2002 November might indicate a weakening or halt of accretion through the inner gap. Because GM Aur had no detectable line emission or continuum excess, we treated it as a WTTS and adopted it as a spectral template for veiling. The spectral type for this star in the literature ranges from K3 to K7. It is certainly warmer than K7 in our spectra, with 
TABLE 1

T TAURI SAMPLE

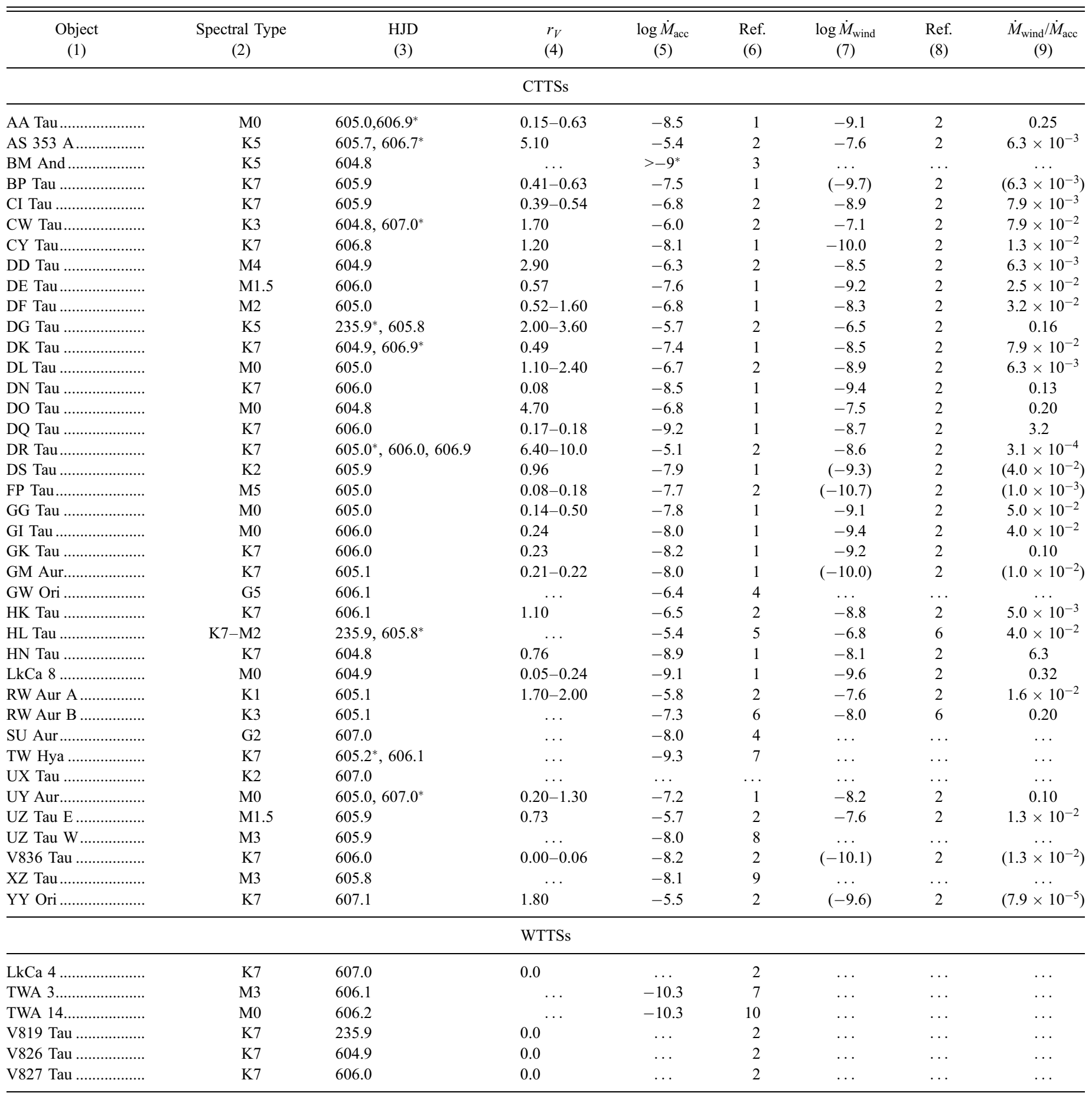

Note.-Col. (2): Spectral types from HEG95, Herbig \& Bell (1988), Reid (2003); col. (3): heliocentric Julian Date (2,452,000+); for multiple observations an asterisk indicates membership in the reference sample; col. (4): Veiling at $5700 \AA$ from HEG95; col. (5): mass accretion rate in $M_{\odot} \mathrm{yr}^{-1}$; col. (7): mass-loss rate in $M_{\odot} \mathrm{yr}^{-1}$; parentheses indicate an upper limit; col. (9): ratio of the mass-loss rate to the mass accretion rate.

REFERENCES.-(1) Gullbring et al. 1998; (2) HEG95; (3) Guenther \& Hessman 1993; (4) Gullbring et al. 2000; (5) Calvet et al. 1994; (6) White \& Hillenbrand 2004; (7) Muzerolle et al. 2000; (8) Hartigan \& Kenyon 2003; (9) Valenti et al. 1993; (10) Muzerolle et al. 2001b.

photospheric features similar to stars classified as $\mathrm{K} 2-\mathrm{K} 3(\mathrm{CW}$ Tau and UX Tau). We also note that two of the other WTTSs, TWA 3 (Hen 3-600A) and TWA 14, have been modeled as possessing extremely small disk accretion rates of $\dot{M}_{\text {acc }}=5 \times$ $10^{-11} M_{\odot} \mathrm{yr}^{-1}$ on the basis of their $\mathrm{H} \alpha$ lines (Muzerolle et al. 2000, 2001b). However, since the derived accretion rates are so small and neither shows significant line or continuum emission in our $1 \mu \mathrm{m}$, spectra we have included them with the WTTSs and adopted TWA 3 as a veiling template.

The final palette of WTTS spectral templates for determination of veiling and residual emission profiles in the CTTS included only five stars, after V826 Tau and TWA 14 were eliminated. Although four of these are identified as $\mathrm{K} 7 / \mathrm{MO}$ in the literature, the relative strengths of their $\mathrm{Ti}, \mathrm{Cr}$, and $\mathrm{Si}$ lines led us to sequence 

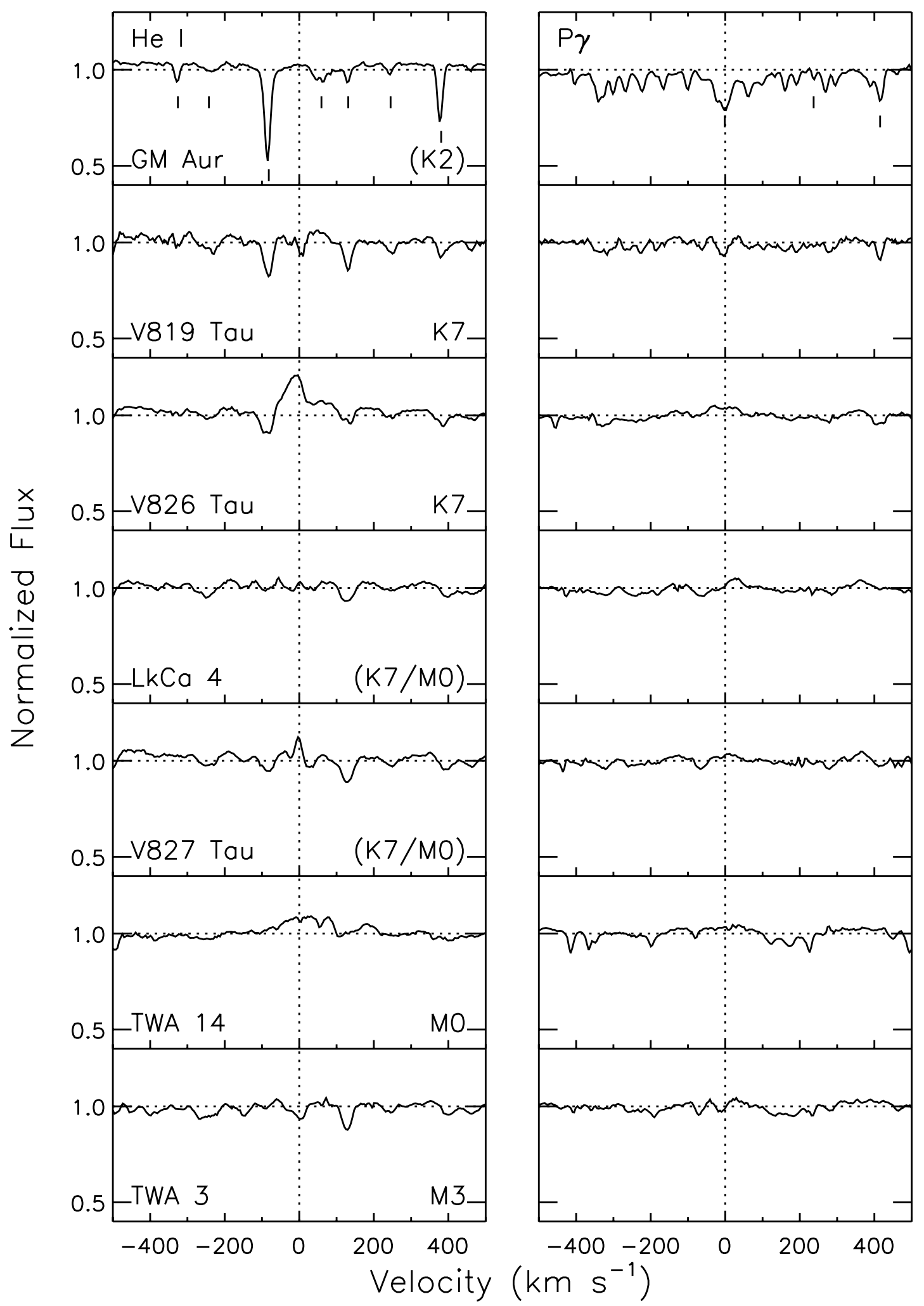

FIg. 1.-Spectral regions of He I $\lambda 10830$ and P $\gamma$ for six WTTSs plus GM Aur (see Table 1). Photospheric features marked with vertical lines are identified in the text, except for the numerous features from $\mathrm{CN} 0-0$ near $\mathrm{P} \gamma$. Ordering is by apparent spectral type, given in parentheses when it differs from published values. Fluxes are normalized to the continuum, velocities measured relative to the stellar photosphere, and spectra are unbinned (i.e., at the instrumental resolution.)

them as shown in Figure 1. These correspond to effective spectral types of $\mathrm{K} 2, \mathrm{~K} 7, \mathrm{~K} 7, \mathrm{~K} 7 / \mathrm{MO}$, and $\mathrm{M} 3$ for veiling determination. With this sequencing our modest number of WTTS templates provided good veiling determinations for the CTTSs with the possible exception of the two solar-type stars, GW Ori and SU Aur.
For these stars we assigned SU Aur $r_{Y}=0$, since its line depths were comparable to our $\mathrm{K} 2$ template after the appropriate rotational broadening, and from there we found that GW Ori, which has a comparable $v \sin i$ to SU Aur, required $r_{Y}=0.3$. In addition there were three stars with a middle to late K spectral type (CI Tau, 
HN Tau, and RW Aur A), which appeared to be intermediate between our K2 or K7 templates, so we took the unorthodox step of creating a synthetic template from averaging the spectra of these two. It provided an excellent match for the relative line depths of these three stars, and we felt the veiling derived with the synthetic template was reliable.

The $Y$-band veiling, $r_{Y}$, for the 38 CTTSs with $\mathrm{He}$ I $\lambda 10830$ and $\mathrm{P} \gamma$ line emission is listed in Table 2. Stars are ordered by veiling, which ranges from $r_{Y}=0-2$. Veiling is first determined independently in each of the two photospheric orders, and then the average value is adopted and applied to the order with the $\mathrm{He}$ I $\lambda 10830$ and $\mathrm{P} \gamma$ lines. Subtraction of the veiled standard from this order yields both residual emission-line profiles for these two lines and a third assessment of the veiling. We found an excellent agreement for veilings determined from the three orders. The error for each veiling measurement is the largest deviation from the adopted value that yields a fit indistinguishable from the best fit, which is typically larger than the dispersion among the orders. When $r_{Y}$ is less than or equal to 0.5 , the veiling error is approximately \pm 0.05 . For larger veilings, the error is approximately \pm 0.1 . We note that the veiling measurements reported here for the six stars that also appear in Edwards et al. (2003) supersede the earlier estimates, which were not evaluated with the same degree of thoroughness.

The process of creating residual profiles results in an improved definition of the emission lines in stars with low veiling, particularly in the line wings. We illustrate this in Figure 2 at $\mathrm{P} \gamma$ for two stars with $r_{Y}=0.1$, AA Tau and UZ Tau W. Note the effect on the line shape, especially how the wings in the residual profiles are more clearly defined when the photospheric features have been removed.

The residual profiles for $\mathrm{P} \gamma$ and $\mathrm{He}$ I $\lambda 10830$ for 38 CTTSs are presented in Figures 3 and 4, respectively. The 39th CTTS, GM Aur, is excluded, since it was not detected in either line. Nine CTTSs were observed more than once, and we show the multiple profiles for $\mathrm{P} \gamma$ and $\mathrm{He}$ I $\lambda 10830$ in Figures 5 and 6, respectively. Most multiple observations are separated by days, except for DG Tau and HL Tau, which are separated by a year. Table 2 presents measurements of the $\mathrm{P} \gamma$ and $\mathrm{He}_{\mathrm{I}} \lambda 10830$ features in the CTTSs including kinematic information and emission and absorption equivalent widths. Among the nine stars with more than one observation, the profiles and their kinematic parameters do not change appreciably, with the exception of AA Tau. For these nine stars, the spectrum included in the reference sample is identified with an asterisk, representing one spectrum for each star, and is used for all statistical analyses in figures and text.

In both the figures and Table 2 the spectra are arranged in order of decreasing $1 \mu \mathrm{m}$ veiling. The table separates the stars into groups corresponding to three levels of veiling: $\operatorname{high}\left(r_{Y} \geq 0.5\right.$; 9 stars $)$, medium $\left(0.3 \leq r_{Y}<0.5 ; 11\right.$ stars $)$ and low $\left(r_{Y} \leq 0.2\right.$; 18 stars). The low-veiling stars are further divided into two subgroups of 9 stars each, according to the width of the $\mathrm{P} \gamma$ feature. Average values for kinematic properties of the profiles in each group are identified. Discussion of the profiles and their kinematic properties follows in subsequent sections.

\section{ACCRETION DIAGNOSTICS: P $\gamma$ AND 1 MICRONVEILING}

Our NIRSPEC data allows us to evaluate two quantities expected to be sensitive to the disk accretion rate: $\mathrm{P} \gamma$ equivalent width and the $1 \mu \mathrm{m}$ veiling, $r_{Y}$. The $\mathrm{P} \gamma$ line emission is predicted to arise primarily in magnetospheric accretion columns (Muzerolle et al. 1998a, 2001a) and the continuum excess from accretion luminosity, e.g., from accretion shocks at the base of magnetic funnel flows (Calvet \& Gullbring 1998). These diagnostics can be used to test magnetospheric accretion models and to establish the extent to which our prime wind diagnostic, $\mathrm{He}$ I $\lambda 10830$, is influenced by accretion. We detect $\mathrm{P} \gamma$ emission in 38 of the 39 CTTSs and $1 \mu \mathrm{m}$ veiling in 31 of the 39 CTTSs. The sole $\mathrm{P} \gamma$ nondetection is GM Aur, which as discussed in the previous section appears to be in a quiescent nonaccreting state, and we treat it as a WTTS for the remainder of this paper (see Fig. 1). In this section we focus on the properties of the $\mathrm{P} \gamma$ emission and its relation to veiling. We discuss the implications of the profile morphology for magnetospheric accretion models in $\S 6.1$ and examine the spectral energy distribution of the veiling and its relation to disk accretion rate in $\S 7$. For the intervening sections we simply use $r_{Y}$ as a quantity likely to be proportional to the instantaneous disk accretion rate.

The $\mathrm{P} \gamma$ profiles for 38 CTTSs are predominantly in emission; none shows blueshifted absorption from a wind, while $24 \%(08$ from the reference sample in Fig. 3: BM And, DR Tau, DS Tau, GI Tau, RW Aur, RW Aur B, SU Aur, and YY Ori, plus AA Tau in Fig. 5) show redshifted absorption below the continuum, suggesting infall of accreting material in a magnetospheric funnel flow. The $\mathrm{P} \gamma$ emission and absorption equivalent widths are listed for each observation in Table 2 . The emission equivalent widths range from a high of $18 \AA$ (AS $353 \mathrm{~A}$ ) to a low of $0.6 \AA$ (CI Tau, $\mathrm{V} 836 \mathrm{Tau}$ ) and the absorption equivalent widths from a high of $1.1 \AA$ (YY Ori) to a low of $0.2 \AA$ (DS Tau and RW Aur A).

We find a remarkably good correspondence between the $\mathrm{P} \gamma$ emission equivalent width and $r_{Y}$, shown in Figure 7 (top). Following standard practice, in the figure the measured equivalent width $W_{\lambda}$ is multiplied by the factor $1+r_{Y}$, which renormalizes it to the photosphere, rather than to the photosphere plus veiling continuum (Basri \& Batalha 1990; Beristain et al. 2001). The figure shows a tight correlation between $\mathrm{P} \gamma$ and $r_{Y}$ for the 31 CTTSs with measurable veiling, with a linear correlation coefficient of 0.97 between the logarithm of the veiling and the logarithm of the veiling-corrected $\mathrm{P} \gamma$ emission. The figure exaggerates the separation in veiling between the 11 CTTSs with the lowest measured veiling, $r_{Y} \sim 0.1$, and the 7 CTTS nondetections, $r_{Y}<0.05$, which were not included in the determination of the correlation coefficient. However, all but one of the non detections have $\mathrm{P} \gamma$ strengths comparable to those with the lowest veilings, so they effectively exhibit the same correlation between $\mathrm{P} \gamma$ emission and veiling. The sole exception to the correlation is TW Hya, which has no detected veiling but robust $\mathrm{P} \gamma$ emission comparable to CTTSs with $r_{Y} \sim 0.3$. The tight relation between $W_{\lambda}(\mathrm{P} \gamma)$ and $r_{Y}$ is reminiscent of, although better defined than, others reported between permitted emission lines and either veiling or disk accretion rates determined from veiling (Basri \& Batalha [1990] for $\mathrm{H} \alpha$; Muzerolle et al. [1998b] for $\mathrm{H} \alpha$ and $\mathrm{P}_{11}$; Muzerolle et al. [1998c] for $\mathrm{Br} \gamma$ and $\mathrm{P} \beta$; Doppmann et al. [2003] for $\mathrm{Br} \gamma$ ).

Similarly, the morphology of the $\mathrm{P} \gamma$ profiles shown in Figure 3 is reminiscent of $\mathrm{P} \beta$ and $\mathrm{Br} \gamma$ profiles (Folha \& Emerson 2001; Muzerolle et al. 1998c), with broad single peaks sometimes accompanied by redshifted absorption below the continuum. The profiles cover a range of line widths and centroids, which we characterize by measuring three kinematic properties: (1) emission centroids, which range from $V_{c}=-150$ to $+29 \mathrm{~km} \mathrm{~s}^{-1}$; (2) maximum blue wing velocities, which range from $V_{\text {bwing }}=$ -400 to $-100 \mathrm{~km} \mathrm{~s}^{-1}$; and (3) line widths measured from the stellar rest velocity to the blueward half-intensity (BHI) point, which range from $V_{\mathrm{BHI}}=-250$ to $-26 \mathrm{~km} \mathrm{~s}^{-1}$. The latter two focus on emission blueward of the stellar rest velocity in order to avoid complications from redshifted absorption. Measurements of $V_{\text {bwing }}, V_{\mathrm{BHI}}$, and $V_{c}$ are plotted against veiling in Figure 8 for all 
TABLE 2

CTTS Veilings and Profiles

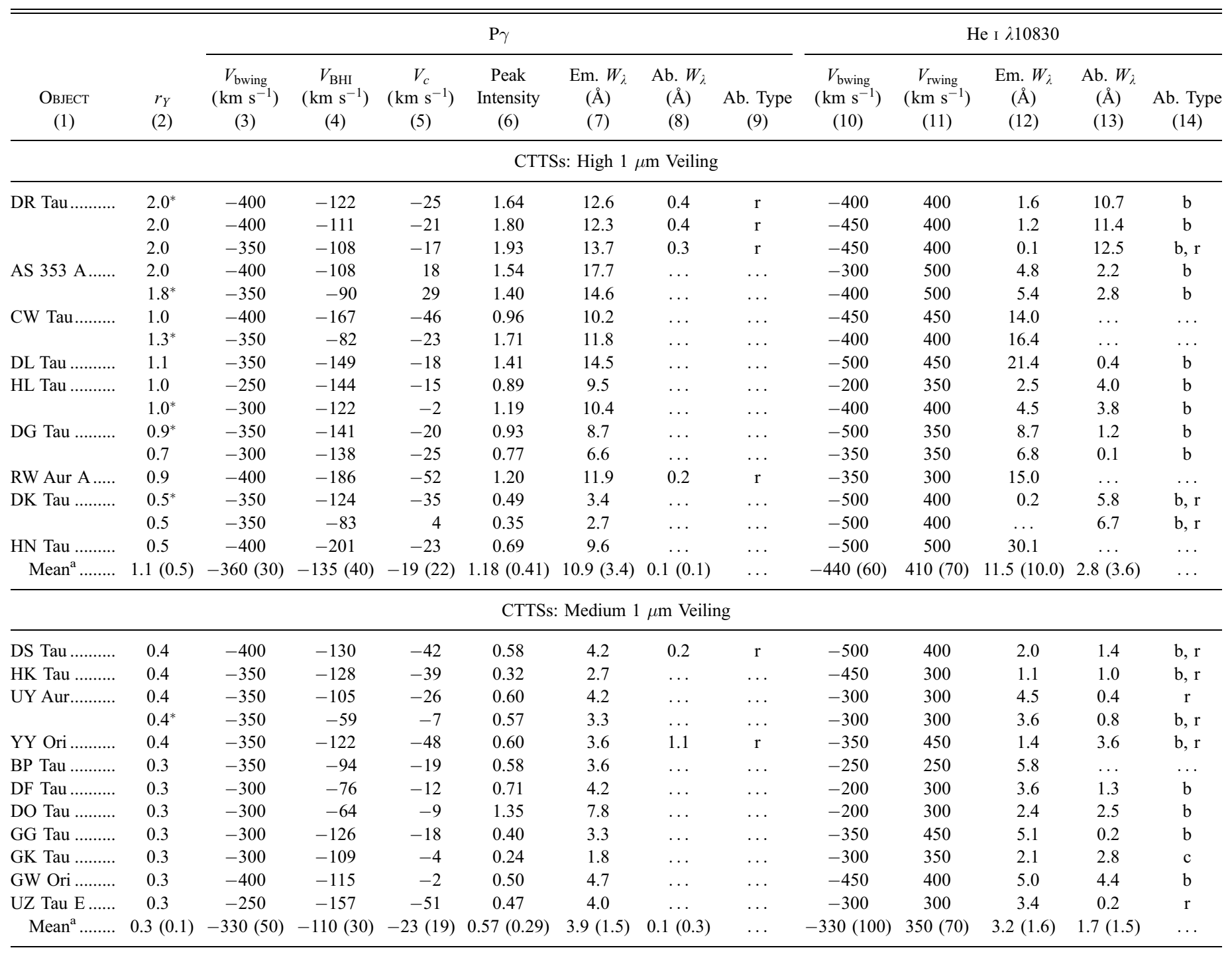

CTTSs: Low $1 \mu \mathrm{m}$ Veiling, Narrow P $\gamma$

\begin{tabular}{|c|c|c|c|c|c|c|c|c|c|c|c|c|c|}
\hline \multirow{2}{*}{ AA Tau............ } & 0.2 & -350 & -94 & -46 & 0.29 & 1.5 & 0.5 & $\mathrm{r}$ & -400 & 400 & 4.4 & 0.6 & $\mathrm{r}$ \\
\hline & $0.1^{*}$ & -300 & -37 & 23 & 0.16 & 0.9 & $\ldots$ & $\ldots$ & -400 & 400 & 1.9 & 2.0 & $\mathrm{r}$ \\
\hline GI Tau ............ & 0.1 & -350 & -45 & -1 & 0.53 & 3.2 & 0.3 & $\mathrm{r}$ & -350 & 400 & 2.2 & 3.7 & $\mathrm{~b}, \mathrm{r}$ \\
\hline DN Tau .......... & 0.0 & -400 & -28 & 4 & 0.24 & 1.1 & $\ldots$ & $\ldots$ & -350 & 350 & 2.1 & 1.7 & $\mathrm{~b}, \mathrm{r}$ \\
\hline DQ Tau .......... & 0.0 & -200 & -42 & 0 & 0.20 & 0.8 & $\ldots$ & $\ldots$ & -450 & 350 & 9.4 & 0.3 & $\mathrm{~b}$ \\
\hline TW Hya ......... & $0.0^{*}$ & -350 & -58 & 0 & 1.71 & 8.8 & $\ldots$ & $\ldots$ & -350 & 350 & 13.6 & 5.3 & $\mathrm{~b}$ \\
\hline XZ Tau............ & 0.0 & -300 & -44 & 1 & 0.21 & 1.0 & $\ldots$ & $\ldots$ & -325 & 250 & 2.8 & 1.2 & $\mathrm{~b}$ \\
\hline Mean $^{\mathrm{a}}$ & $0.0(0.0)$ & $-290(90)$ & $-40(10)$ & $2(8)$ & $0.42(0.50)$ & $2.2(2.6)$ & $0.0(0.1)$ & $\ldots$ & $-320(90)$ & $340(50)$ & $4.2(4.2)$ & $2.3(1.5)$ & $\cdots$ \\
\hline
\end{tabular}

CTTSs: Low $1 \mu \mathrm{m}$ Veiling, Broad P $\gamma$

\begin{tabular}{|c|c|c|c|c|c|c|c|c|c|c|c|c|c|}
\hline CI Tau ................ & 0.2 & $\ldots$ & $\ldots$ & $\ldots$ & 0.06 & 0.6 & $\ldots$ & $\ldots$ & -200 & 350 & 0.1 & 2.2 & $\mathrm{~b}, \mathrm{r}$ \\
\hline DE Tau .............. & 0.2 & -250 & -128 & -12 & 0.28 & 2.4 & $\ldots$ & $\ldots$ & -350 & 350 & 1.9 & 0.7 & $\mathrm{~b}$ \\
\hline 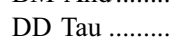 & 0.1 & -300 & -106 & -49 & 0.38 & 2.2 & $\ldots$ & $\ldots$ & -150 & 250 & 0.5 & 0.2 & $\mathrm{~b}$ \\
\hline RW Aur B ..... & 0.1 & -150 & -85 & -9 & 0.14 & 0.7 & 0.5 & $\mathrm{r}$ & -300 & 400 & 1.5 & 2.8 & $\mathrm{r}$ \\
\hline UZ Tau W..... & 0.1 & -300 & -91 & -44 & 0.25 & 1.4 & $\ldots$ & $\ldots$ & -250 & 250 & 0.3 & 0.8 & $\mathrm{~b}, \mathrm{r}$ \\
\hline SU Aur............ & 0.0 & -400 & -199 & -120 & 0.17 & 1.4 & 0.6 & $\mathrm{r}$ & -450 & 200 & 0.1 & 2.1 & $\mathrm{~b}, \mathrm{r}$ \\
\hline UX Tau ........... & 0.0 & $\ldots$ & $\ldots$ & $\ldots$ & 0.09 & 1.3 & $\ldots$ & $\ldots$ & -400 & 300 & 2.3 & 2.0 & $\mathrm{c}$ \\
\hline
\end{tabular}

Notes.-Col. (2): An asterisk following $r_{Y}$ identifies the observation chosen for the reference sample. Col. (6): Peak emission intensity in units of the continuum. Cols. (8), (13): Equivalent width of all absorption below the continuum. Cols. (9), (14): Type of absorption: blue (b); central (c); red (r).

${ }^{a}$ Mean profile parameters for the reference sample from each veiling group; standard deviation in parentheses. 


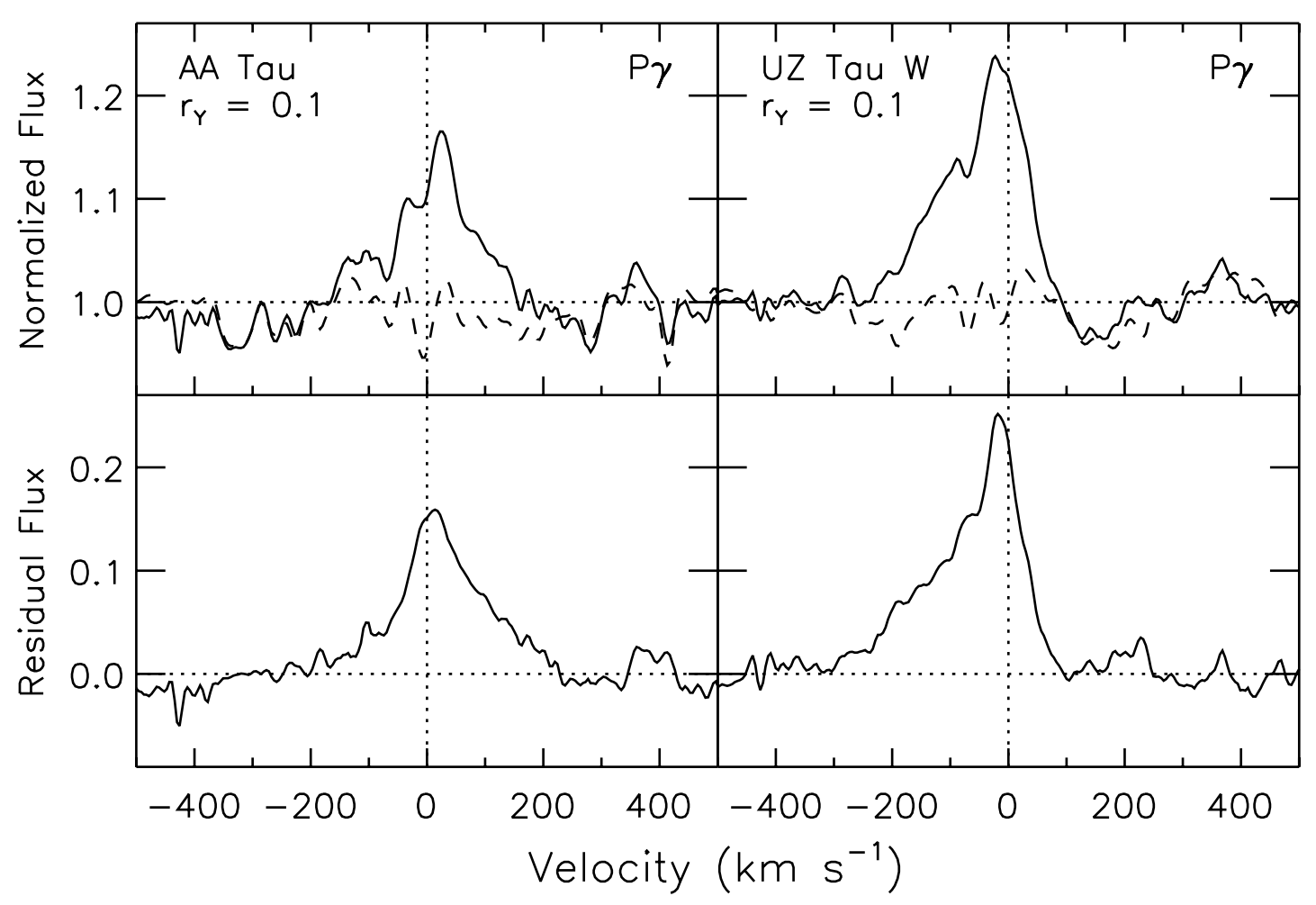

FIG. 2.-Demonstration of residual profile determination at $\mathrm{P} \gamma$ in AA Tau and UZ Tau W. Top: Fluxes relative to the continuum for the CTTSs (solid line) and for the veiled template (dashed line). The resultant residual profiles after subtraction of the template from the star are shown in the lower row. Templates are V819 Tau (K7) for AA Tau and TWA 3 (M3) for UZ Tau W. Velocities are relative to the stellar photosphere.

but two stars in the reference sample and listed in Table 2 for all observations. The two stars omitted in the figure (CI Tau, UX Tau) have such weak and amorphous $\mathrm{P} \gamma$ emission that kinematic features could not be reliably established.

The kinematic data for $\mathrm{P} \gamma$ suggest a relation that has not previously been reported: a connection between $\mathrm{P} \gamma$ profile morphology and veiling. We illustrate it more directly in Figure 9, in which we superpose $\mathrm{P} \gamma$ profiles in groups segregated by veiling. The top panel displays profiles for 9 CTTSs with high veiling $\left(r_{Y} \geq 0.5\right)$, the second panel for 11 CTTSs with medium veiling $\left(0.3 \leq r_{Y}<\right.$ $0.5)$, and last two panels for 18 CTTSs with low or absent veiling $\left(r_{Y} \leq 0.2\right)$. The low-veiling group is divided into two subcategories according to the width of their $\mathrm{P} \gamma$ profiles: 9 CTTSs in the third panel have narrow $\mathrm{P} \gamma$ lines with $V_{\mathrm{BHI}}<60 \mathrm{~km} \mathrm{~s}^{-1}$ and 9 CTTSs in the fourth panel have $\mathrm{P} \gamma$ lines in excess of this width.

The trend in profile morphology with veiling is exhibited by the 29 stars in the top three panels (i.e., excluding the 9 low veiling stars with broad $\mathrm{P} \gamma$ profiles, which are discussed below). For these 29 stars, $\mathrm{P} \gamma$ profiles are normalized to their peak intensities (given for each star in Table 2) to facilitate comparison of profile morphology independent of line strength. Among these 29 stars, the mean line width blueward of the stellar velocity $V_{\mathrm{BHI}}$ decreases from 135 to $40 \mathrm{~km} \mathrm{~s}^{-1}$ among the three veiling groups, while the mean centroid velocity decreases from $V_{c} \sim 20$ to $\sim 0 \mathrm{~km} \mathrm{~s}^{-1}$. Remarkably, those in the low-veiling subgroup with narrow $\mathrm{P} \gamma\left(V_{\mathrm{BHI}} \leq 60 \mathrm{~km} \mathrm{~s}^{-1}\right)$ all have rather similar profiles after normalization, showing central peaks with an average $V_{\mathrm{BHI}}=-40 \pm 10 \mathrm{~km} \mathrm{~s}^{-1}$, an average $V_{c}=2 \pm 8 \mathrm{~km} \mathrm{~s}^{-1}$. Most also have a two-component structure suggesting a strong narrow core and a weak broad base. When we compute an average $\mathrm{P} \gamma$ profile for these nine low-veiling stars, it is well fit with two Gaussians, one a narrow component centered at $-2 \mathrm{~km} \mathrm{~s}^{-1}$ with an FWHM of $52 \mathrm{~km} \mathrm{~s}^{-1}$ and the other a broad component centered at $+6 \mathrm{~km} \mathrm{~s}^{-1}$ with an FWHM of $176 \mathrm{~km} \mathrm{~s}^{-1}$.

Although the trend in $\mathrm{P} \gamma$ profile morphology to decrease in width and centroid velocity with decreasing veiling is suggestive, it does not include nine stars with low-veiling (24\% of the sample). The separation of the low-veiling stars into two groups based on $\mathrm{P} \gamma$ line width was effected in order to illustrate the remarkable uniformity for half of the low-veiling profiles. The other nine low-veiling stars with broad $\mathrm{P} \gamma$, superposed in Figure 9 (bottom), show an unusual diversity in their morphology. Three (BM And, CI Tau, UX Tau) have broad amorphous emission and low peak intensity ( $<10 \%$ of the continuum flux). Two (BM And, SU Aur) have exceptionally deep and broad redshifted subcontinuum absorption penetrating to $50 \%$ of the $1 \mu \mathrm{m}$ continuum with emission peaking considerably blueward of the other stars $\left(V_{c}<-120 \mathrm{~km} \mathrm{~s}^{-1}\right)$. Along with these four stars that are completely unlike the rest of the sample, the additional five stars in this subgroup (DE Tau, DD Tau, FP Tau, RW Aur B, and UZ Tau W) are more typical but are included here because their breadths and blueshifts are larger than those with uniform narrow $\mathrm{P} \gamma$ profiles. (We note that unlike the other superposed profiles in Fig. 9, the nine stars in this subgroup are plotted in residual intensity units, reflecting their true height above the stellar continuum, rather than normalized to peak intensity, to avoid misrepresenting the amorphous profiles with very low peak intensities.)

In summary, our key findings for $\mathrm{P} \gamma$ and veiling are (1) a robust correlation between emission equivalent width and veiling; (2) a remarkably consistent profile morphology among half of the stars with low veiling showing a two component structure with narrow centered cores and a weak broad base; and (3) a progression in profile morphology with veiling for a sizeable fraction, but not all, of the sample. Systematic variations with veiling of line width and centroid velocity have not to our knowledge been 


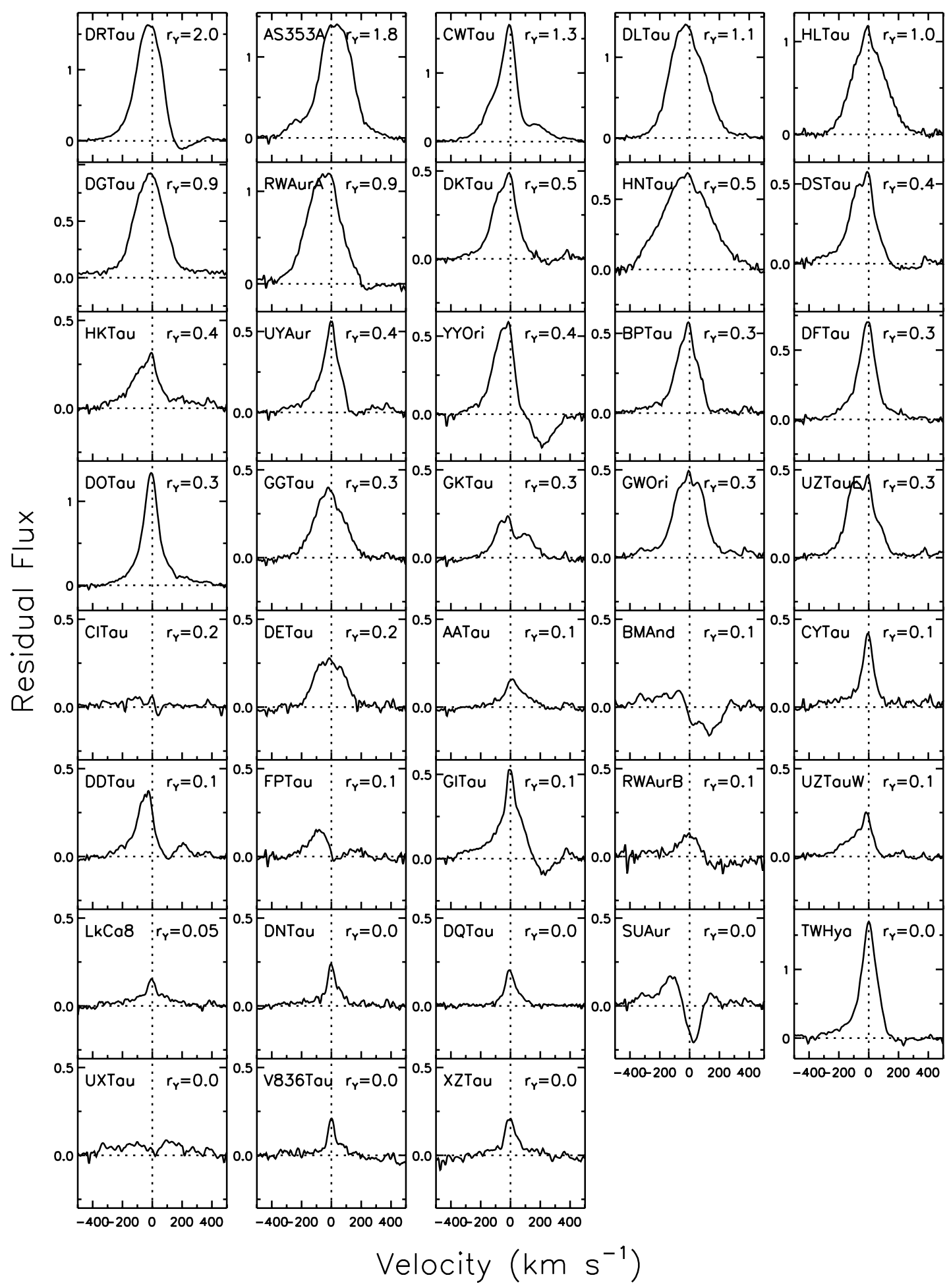

Fig. 3.-Residual $\mathrm{P} \gamma$ profiles from the reference sample for 38 CTTSs (listed in Table 1), ordered by decreasing $1 \mu \mathrm{m}$ veiling $r_{Y}$. Velocities are relative to the stellar photosphere and spectra are plotted with 3 pixel binning.

previously reported for hydrogen lines in CTTSs. We discuss the implication of the veiling dependence of $\mathrm{P} \gamma$ profiles for magnetospheric formation in $\S 6.1$.

\section{WIND DIAGNOSTIC: He I $\lambda 10830$}

Our NIRSPEC survey probes the innermost wind region in accreting stars with the He r $\lambda 10830$ line. The uniqueness of this probe derives from the metastability of its lower level $\left(2 s^{3} S\right)$, which, although energetically far above $(20 \mathrm{eV})$ the singlet ground state, is radiatively isolated from it. Whether this metastable level is populated by recombination and cascade or by collisional excitation from the ground state, it will become significantly populated relative to other excited levels owing to its weak deexcitation rate via collisions to singlet states, making it an ideal candidate to form an absorption line. This absorption is essentially a resonantscattering process, since the $\lambda 10830$ transition $\left(2 p^{3} P^{0}-2 s^{3} S\right)$ is the only permitted radiative transition from its upper state to a lower state and the electron density is unlikely to be so high 


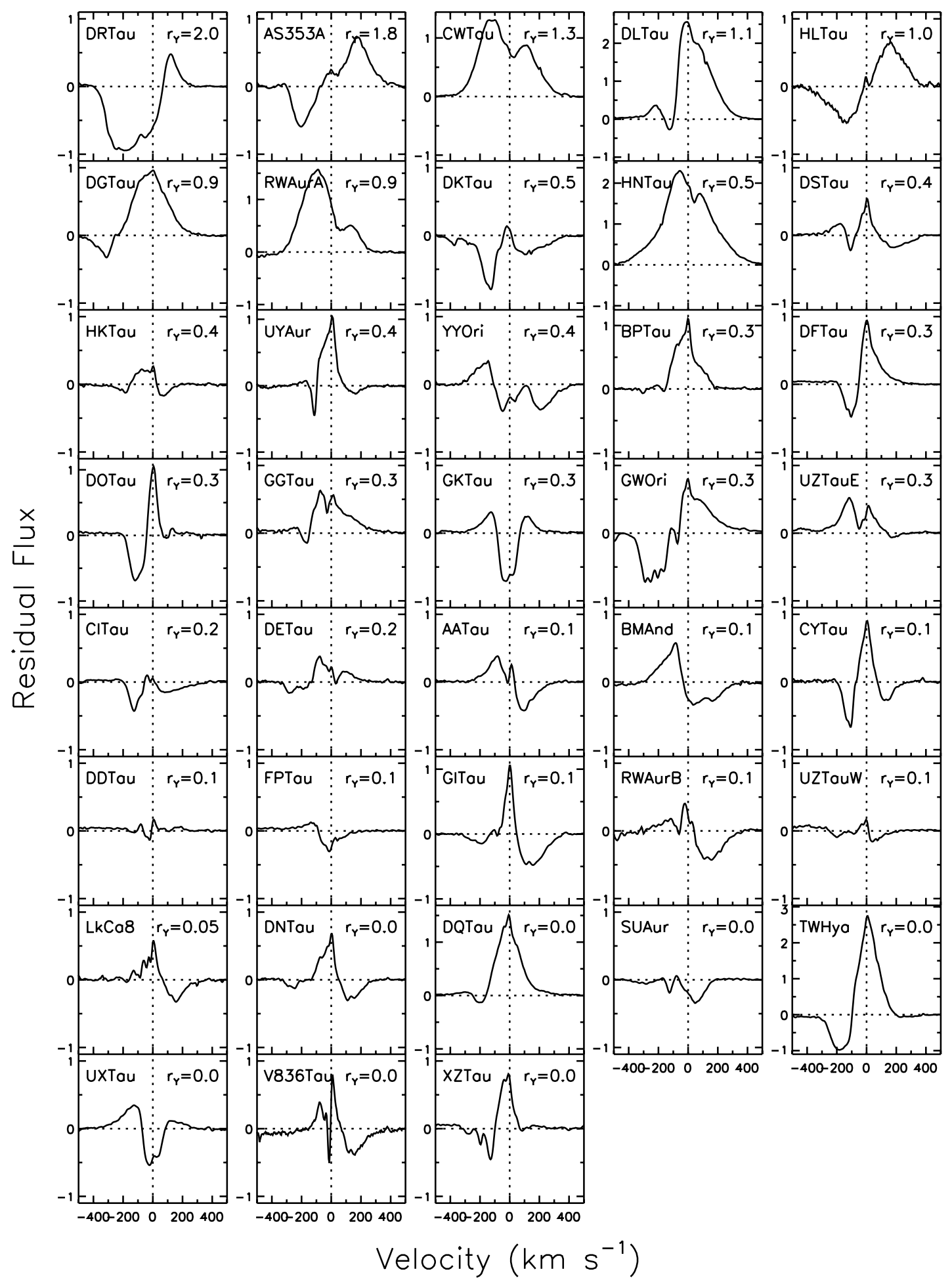

FIg. 4.-Same as Fig. 3, but for residual He I $\lambda 10830$ profiles.

as to cause collisional excitation or deexcitation. This sets up an ideal situation to probe outflowing gas in absorption, provided the conditions are right to excite helium $20 \mathrm{eV}$ above the ground state.

We find He I $\lambda 10830$ features in 38 of the 39 CTTSs in our sample; the exception is GM Aur (see Fig. 1), suspected to be in a quiescent nonaccreting state. Line profiles for each star, ordered by decreasing $r_{Y}$, are shown in Figure 4, and additional observations of the nine stars with multiple observations are in Figure 6. Equivalent widths and kinematic properties are listed in
Table 2, sorted by four groups corresponding to high, medium, and low veiling, with the 18 low-veiling objects additionally split two kinematic subgroups based on $\mathrm{P} \gamma$ line widths, $V_{\mathrm{BHI}}$.

The predicted sensitivity of $\mathrm{He}_{\mathrm{I}} \lambda 10830$ to absorption is born out by our observations, where absorption below the $1 \mu \mathrm{m}$ continuum is seen in 34 of the 38 stars $(89 \%)$. Although blueshifted subcontinuum absorption from outflowing gas is most common ( 27 of 38 stars; $71 \%$ ) redshifted subcontinuum absorption from infalling gas is also frequent (18 out of 38 stars; 47\%). In 2 stars ( $5 \%$; GK Tau, UX Tau) the subcontinuum absorption is centered 

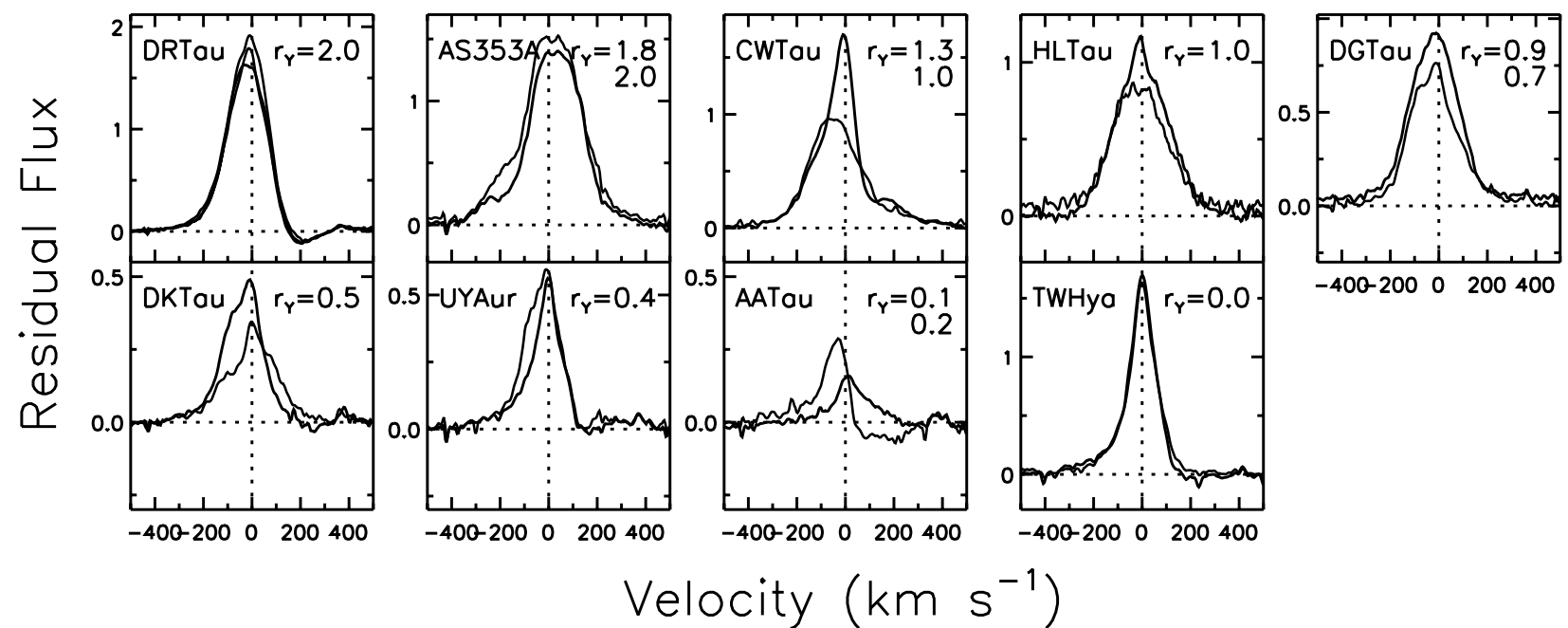

Velocity $\left(\mathrm{km} \mathrm{s}^{-1}\right)$

FIG. 5.-Multiple observations of $\mathrm{P} \gamma$ residual profiles for nine CTTSs. For each object the spectrum from the reference sample is denoted by a heavier line.

on the stellar rest velocity and is flanked by emission on both sides. Our identification of the presence of blue, red, or centered subcontinuum absorption in each profile is listed in column (14) of Table 2, marked by the letters b, r, and c. Of the four stars with no subcontinuum He I $\lambda 10830$ absorption (CW Tau, RW Aur A, HN Tau, BP Tau) the profiles have asymmetries suggesting either redward or blueward absorption that does not penetrate the $1 \mu \mathrm{m}$ continuum.

Because subcontinuum absorption is often a sizeable contributor to the He I $\lambda 10830$ line, a comparison between emission equivalent width and veiling, which yielded a well-defined relationship for $\mathrm{P} \gamma$, is not meaningful for $\mathrm{He}_{\mathrm{I}} \lambda 10830$. While the emission equivalent width above the continuum ranges from $W_{\lambda}=0$ to $30.1 \AA$, the absorption equivalent width below the continuum in turn ranges from $W_{\lambda}=0$ to $10.7 \AA$, so that a strong helium feature may have a large positive or negative equivalent width or may have a net equivalent width close to zero. To examine the dependence of the strength of He I $\lambda 10830$ with veiling, we define a helium activity index as the sum of the absolute value of the equivalent width of the emission above the continuum $(E)$ and the absorption below the continuum $(A)$. This activity index $(E+A)$ ranges from $\sim 1-30$ and is plotted against
$r_{Y}$ in Figure 7 (bottom). It shows a trend of increasing with veiling, corresponding roughly to that seen for $\mathrm{P} \gamma$, although with considerably more scatter. We have separately indicated the helium profiles that are primarily in emission $(E-A / E+A>0.5)$, since the activity index for these stars is dominated by the emission equivalent width, which is directly analogous with the relation found for $\mathrm{P} \gamma$. The observed trend relating the helium activity index and veiling indicates that helium excitation is correlated with disk accretion rate.

In order to appreciate the full diversity of helium profiles, we have arranged them into a scheme based on a combination of both veiling level and profile structure. This categorization is shown in Figure 10, in which superposed He $\mathrm{I} \lambda 10830$ profiles are first grouped by the same four categories used for comparing $\mathrm{P} \gamma$ profiles in Figure 9 based on veiling and the $\mathrm{P} \gamma$ line width (four horizontal rows), and then further sorted into three morphological classes (three vertical columns). All helium profiles are in the continuum-normalized units of Figure 4, with the exception of TW Hya, which was rescaled to half its peak intensity in order to facilitate comparison with the other profiles in its category.

The three morphological groups portrayed in Figure 10 are as follows: The left column contains 14 stars with profiles that fall
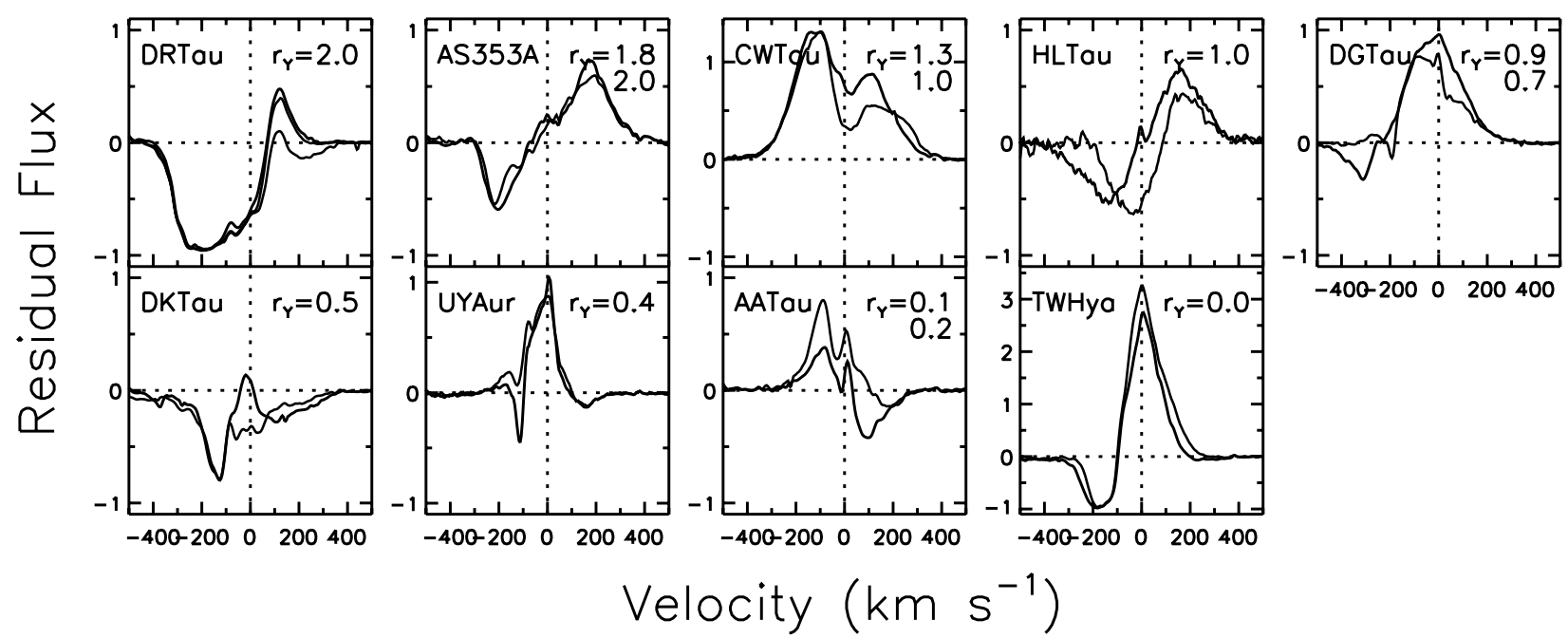

Velocity $\left(\mathrm{km} \mathrm{s}^{-1}\right)$

FIG. 6. - Same as Fig. 5, but for He I $\lambda 10830$ residual profiles. 

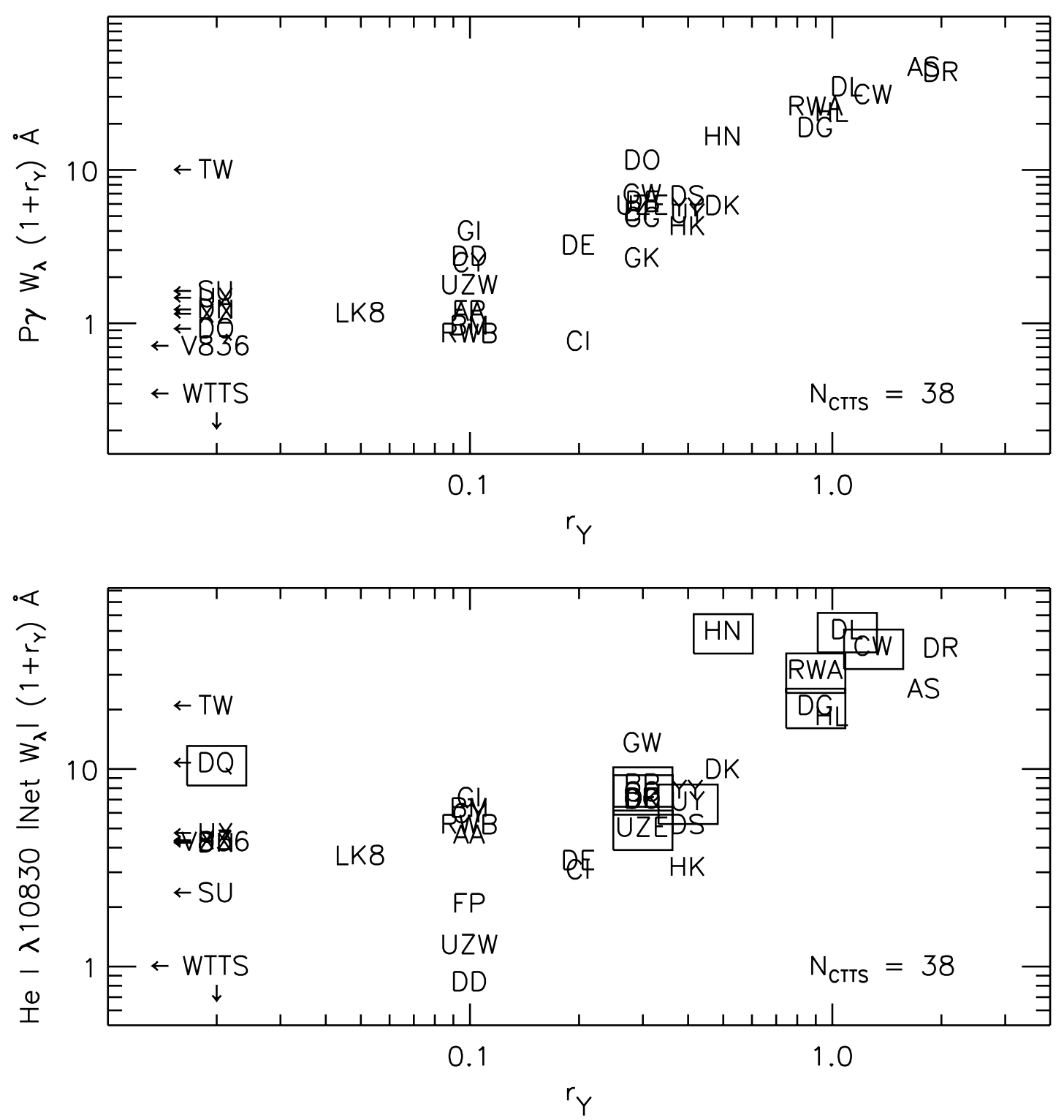

FIG. 7.-Top: Veiling-corrected P $\gamma$ emission equivalent width vs. $Y$-band veiling for 38 CTTSs from the reference sample. Bottom: Veiling-corrected He I $\lambda 10830$ activity index (sum of absolute values of emission plus absorption equivalent widths) vs. $Y$-band veiling for the same stars. In the He I $\lambda 10830$ panel a box around the name of the star indicates its equivalent width is primarily in emission (see text). Veiling nondetections are shown at $r_{Y}=0.02$ for clarity. The location of the six WTTSs plus GM Aur are also indicated.

into the general category of "P Cygni-like," with subcontinuum absorption that is blueward of all or most of the emission and with no redshifted absorption below the continuum. The middle column contains 18 stars with profiles that do have redshifted absorption below the continuum, most of which (13 stars) also show subcontinuum blueshifted absorption. The profiles in the right column are from the 6 objects with either central absorption (2 stars) or no subcontinuum absorption (4 stars).

The ensemble of $\mathrm{He}$ I $\lambda 10830$ profiles in Figure 10 illustrates that each of the three general profile types (blueshifted subcontinuum absorption, both blueshifted and redshifted subcontinuum absorption, and central or no subcontinuum absorption) can be found at all veiling levels. However, there is a tendency for broad P Cygni-like profiles to be more common among stars of high veiling (5 out of 9 stars), while narrow emission coupled with both blueshifted and redshifted absorption is more common among stars with low veiling (12 out of 18 stars). Although our time coverage is limited, among the 9 stars with more than one observation we do not see any stars shifting among these profile categories (see Fig. 6), suggesting that the profile categories we observe are not primarily due to time variable behavior among the CTTSs but are intrinsic to each star.

Examination of the superposed profiles in Figure 10 also shows that the kinematic structure of the subcontinuum blueshifted absorption component is more diverse than that of the red absorption. We discuss the implications of this diversity for wind formation in $\S 6.2$ and present here a table with basic properties of the blueshifted absorption in Table 3 . This includes the equivalent width of the blue absorption below the continuum, the continuum penetration depth, the full velocity extent of the blue absorption and the corresponding minimum and maximum velocity relative to the stellar rest velocity. At one extreme is an object like DR Tau, in which the $\mathrm{He}_{\mathrm{I}} \lambda 10830$ line is almost entirely in absorption, with a breadth of nearly $500 \mathrm{~km} \mathrm{~s}^{-1}$, extending from -400 to $+70 \mathrm{~km} \mathrm{~s}^{-1}$ relative to the star and absorbing $95 \%$ of the $1 \mu \mathrm{m}$ continuum over most of that velocity interval. At 


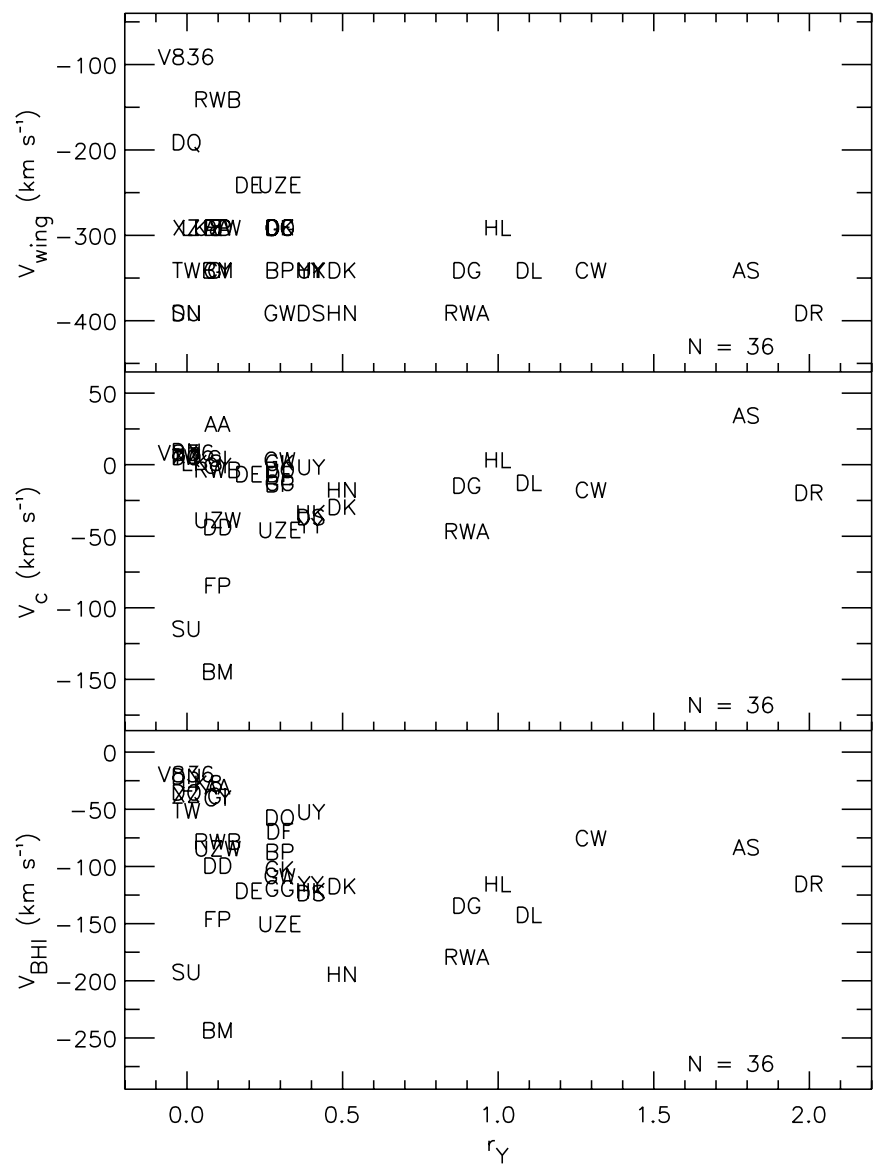

FIG. 8.-Kinematic parameters $V_{\text {bwing }}, V_{\mathrm{BHI}}$, and $V_{c}$ for $\mathrm{P} \gamma$ profiles plotted against veiling.

another extreme, an object like V836 Tau has helium primarily in emission and the narrow blueshifted absorption extends from only -30 to $0 \mathrm{~km} \mathrm{~s}^{-1}$ relative to the star, cutting through the emission feature and penetrating the continuum to a depth of $30 \%$.

In summary, the prevalence in accreting $\mathrm{T}$ Tauri stars of blueshifted He I $\lambda 10830$ absorption below the continuum demonstrates the ubiquity of accretion powered winds arising in the immediate vicinity of the star. However, the diversity of profile morphologies suggests that the nature of the winds are not identical in all of these accreting stars, a topic we return to in $\S 6.2$. We also find that $\mathrm{He}_{\mathrm{I}} \lambda 10830$ is sensitive to infalling gas in magnetospheric accretion flows, as discussed further in $\S 6.1$.

\section{CLASS I SOURCES}

In addition to the survey of 39 CTTSs and 6 WTTSs whose accretion properties are well documented from previous studies, we acquired NIRSPEC data for three young stellar objects, identified in Table 4, whose spectral energy distributions place them as Class I or borderline Class I/II objects. Two of them, SVS 13 and IRAS $04303+2240$, have disk accretion rates comparable to those of the most active Class II CTTSs, $\dot{M}_{\mathrm{acc}} \sim 10^{-6} M_{\odot} \mathrm{yr}^{-1}$ and are the driving sources for Herbig-Haro (HH) objects/jets and molecular outflows (references given in Table 4). The third, IRAS $04248+2612$, is considerably less active, with disk accretion and wind mass-loss rates several orders of magnitude smaller, comparable to a typical CTTS (White \& Hillenbrand 2004).

The faintness of these embedded sources resulted in lower $\mathrm{S} / \mathrm{N}$ spectra than for the CTTSs, for which integration times of $300 \mathrm{~s}$ for IRAS $04303+2240$ and $1200 \mathrm{~s}$ for SVS 13 and IRAS $04248+$ 2612 yielded $\mathrm{S} / \mathrm{N}$ of 60,60 , and 20 , respectively). For IRAS $04248+2612$, the M5.5 photospheric features showed no evidence of veiling. The other two objects have strong emission lines at the location of all the photospheric features, preventing a direct measure of the veiling. For them we estimated the veiling based on the relation between $\mathrm{P} \gamma$ and $r_{Y}$ that we found for the CTTSs. If they follow the same relation, their $\mathrm{P} \gamma$ equivalent widths indicate $r_{Y} \sim$ 1.8 for IRAS $04303+2240$ and $r_{Y} \sim 0.3$ for SVS 13 .

The residual $\mathrm{He}$ I $\lambda 10830$ and $\mathrm{P} \gamma$ profiles for these three stars are shown in Figure 11, and their line properties are given in Table 5. Overall the profile morphologies for both $\mathrm{P} \gamma$ and $\mathrm{He} \mathrm{I}$ $\lambda 10830$ are similar in character to those found among the CTTSs, with the exception of the $\mathrm{P} \gamma$ profile of the low accretion rate object IRAS 04248+2612, which has a double- rather than single-peaked structure. All three He I $\lambda 10830$ lines show evidence of winds in the form of blueshifted absorption, penetrating the continuum in SVS 13 and IRAS $04248+2612$ but not in IRAS $04303+2240$. The He I $\lambda 10830$ profile for SVS 13 is very similar to that of the high accretion rate CTTS DR Tau, in that both are almost entirely in absorption, which is very broad and very deep, extending from the stellar rest velocity to almost $400 \mathrm{~km} \mathrm{~s}^{-1}$.

\section{DISCUSSION OF LINE PROFILES}

Our survey of He I $\lambda 10830$ and $\mathrm{P} \gamma$ lines in CTTS and three Class I sources presents a rich new array of kinematic diagnostics of the star-disk interface region in accreting stars. In this section we discuss how the profile morphology for each of these lines gives insight into magnetospheric accretion flows and accretion powered winds.

\subsection{Magnetospheric Contributions to $\mathrm{P} \gamma$ and $\mathrm{He}$ I $\lambda 10830$}

The presence of magnetospheric accretion flows in classical $\mathrm{T}$ Tauri stars is inferred from general similarities between observed and model profiles, particularly for hydrogen lines of modest opacity (Muzerolle et al. 2001a; Hartmann et al. 1994; Edwards et al. 1994). In the radiative transfer models, line emission arises along the full length of an accretion column characterized by a fairly uniform temperature, connecting the truncated disk to the stellar photosphere. Hydrogen profile characteristics reproduced in the models include broad single-peaked emission with a blue asymmetry and the appearance of redshifted inverse P Cygni (IPC) absorption when the optical depth is not too high and the inclination is favorable (i.e., when the line-of-sight passes through both the infalling gas and the hot accretion shock). In our NIRSPEC data, the presence of redshifted IPC absorption in $\mathrm{P} \gamma$ (9/38 CTTSs) and He I $\lambda 10830$ (18/38 CTTSs) profiles implies that each of these lines probes magnetospheric funnel flows. However, some unexpected profile characteristics pose new challenges to our understanding of the magnetospheric accretion process.

A hydrogen line such as $\mathrm{P} \gamma$ is expected to be formed primarily in the accretion flow, and taken in aggregate, our observations appear to support this expectation. The profiles are single peaked with a range of line widths ( $V_{\mathrm{BHI}}$ from -250 to $\left.-26 \mathrm{~km} \mathrm{~s}^{-1}\right)$, a tendency toward blueward centroids ( $V_{c}$ from -150 to $29 \mathrm{~km} \mathrm{~s}^{-1}$ ), and an IPC absorption frequency of $24 \%$. However, the extremes in line widths and centroid velocities and their progression with veiling are not predicted by existing models.

To quantify the difference between observed and predicted profiles, in Figure 12 we compare distributions of their kinematic parameters $V_{\mathrm{BHI}}$ and $V_{c}$. The kinematic parameters for the data are separately plotted for $\mathrm{P} \gamma$ profiles in the high-, middle-, and 


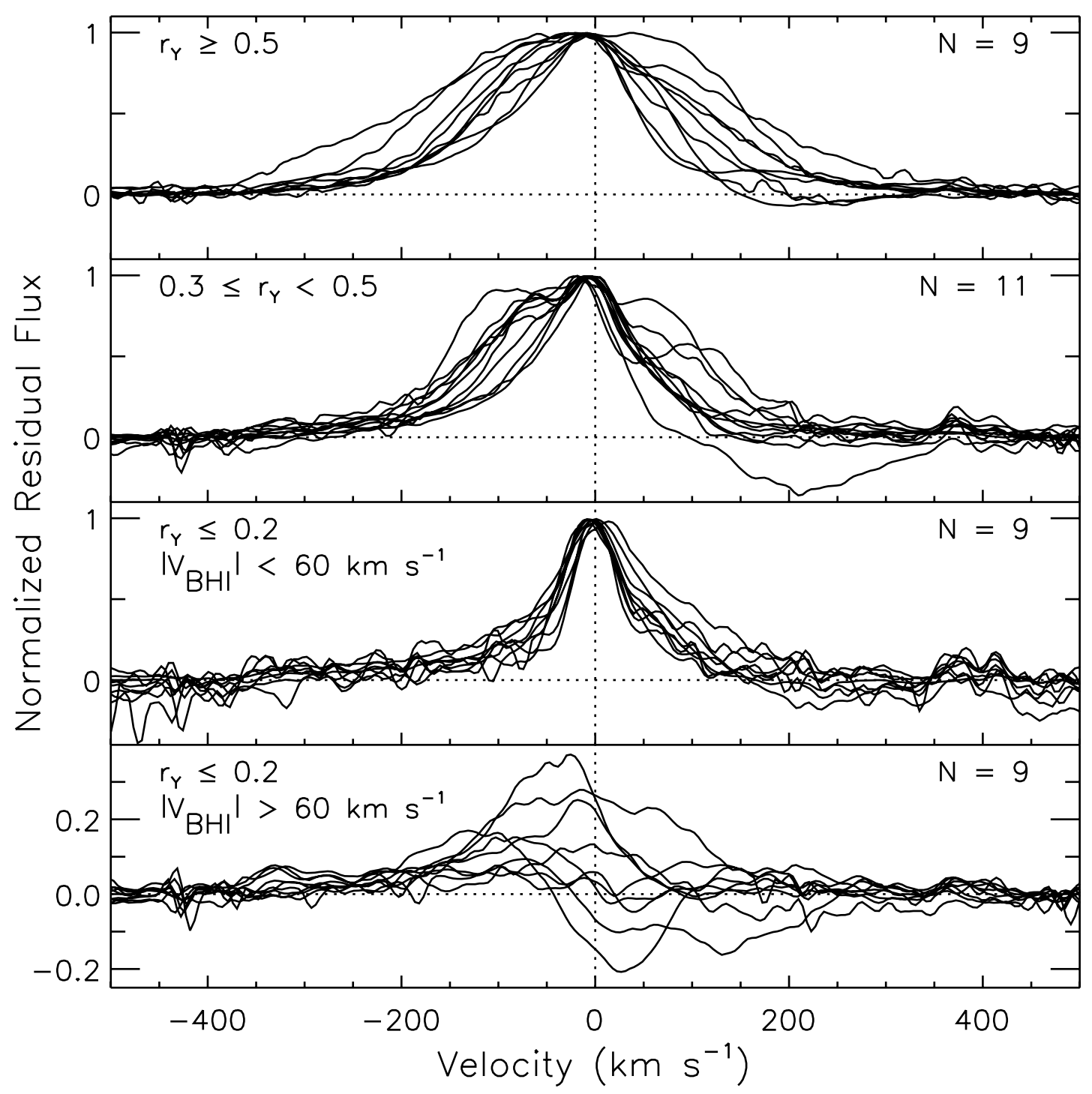

FIG. 9.- Superposed $\mathrm{P} \gamma$ residual profiles from the reference sample grouped by veiling, with the lowest veiling group subdivided into narrow and wide profiles (third and fourth panels). In the top three panels profiles are normalized to their peak intensities. In the bottom panel the residual profiles are not normalized in order not to distort the very weak amorphous emission characterizing three stars.

low-veiling groups described in $\S 3$. Corresponding distributions for the theoretical profiles from magnetospheric accretion models (Muzerolle et al. 2001a) are shown in the bottom panel. The theoretical parameters are measured from the online database of $\mathrm{P} \beta$ profiles predicted to arise under a variety of mass accretion rates and disk truncation radii. ${ }^{6}$ We selected only those profiles considered by Muzerolle to be appropriate combinations of gas temperature and accretion rate, which are $T=6000$ or $7000 \mathrm{~K}$ at $\dot{M}=10^{-6} M_{\odot} \mathrm{yr}^{-1}, T=7000$ or $8000 \mathrm{~K}$ at $\dot{M}=10^{-7} M_{\odot} \mathrm{yr}^{-1}$, $T=8000$ or $10,000 \mathrm{~K}$ at $\dot{M}=10^{-8} M_{\odot} \mathrm{yr}^{-1}$, or $T=10,000$ or $12,000 \mathrm{~K}$ at $\dot{M}=10^{-9} M_{\odot} \mathrm{yr}^{-1}$. Following these constraints, we extracted 128 of the available 272 stark broadened model $\mathrm{P} \beta$ profiles, covering disk truncation radii from $\sim 2$ to $6 R_{*}$ seen from four viewing angles and evaluated their $V_{\mathrm{BHI}}$ and $V_{c}$. To facilitate comparison between the theoretical and observed distributions, we rescaled the $V_{\mathrm{BHI}}$ and $V_{c}$ distributions measured from the models to correspond to the appropriate weighting for a random selection of orientation angles.

\footnotetext{
${ }^{6}$ Available at http://cfa-www.harvard.edu/cfa/youngstars/models/ magnetospheric_ models.html.
}

Although the model and observed profiles span similar ranges of $V_{\mathrm{BHI}}$ and $V_{c}$ for many of the stars, some observed profiles show kinematic properties outside the range of the models. At high veiling two of the nine stars have $V_{\mathrm{BHI}} \sim 50 \mathrm{~km} \mathrm{~s}^{-1}$ higher than the models. More significantly, at low veiling many stars have $V_{\mathrm{BHI}}<50 \mathrm{~km} \mathrm{~s}^{-1}$ and $V_{c}<10 \mathrm{~km} \mathrm{~s}^{-1}$, narrower and more centered than predicted for magnetospheric accretion flows. Moreover, as discussed in $\S 3$, these narrow and centered $\mathrm{P} \gamma$ kinematic properties (Fig. 9, third panel) are actually from the narrow component of profiles with a two-component (narrow and broad) structure.

The $\mathrm{P} \gamma$ narrow component is reminiscent of a similar feature in many metallic emission lines in CTTSs, usually attributed to formation in a stationary accretion shock at the footpoint of the funnel flow (Batalha et al. 1996; Johns-Krull \& Basri 1997; Alencar \& Basri 2000; Najita et al. 2000). For example, the FWHM of the narrow component at $\mathrm{He}$ I $\lambda 5876$ from the study of BEK01, which overlaps significantly with the stars studied here, is $47 \pm 7 \mathrm{~km} \mathrm{~s}^{-1}$, in comparison to that for the average $\mathrm{P} \gamma$ value of $\sim 52 \mathrm{~km} \mathrm{~s}^{-1}$. In CTTS metallic lines the proportional contribution of narrow and broad component emission is sensitive to 


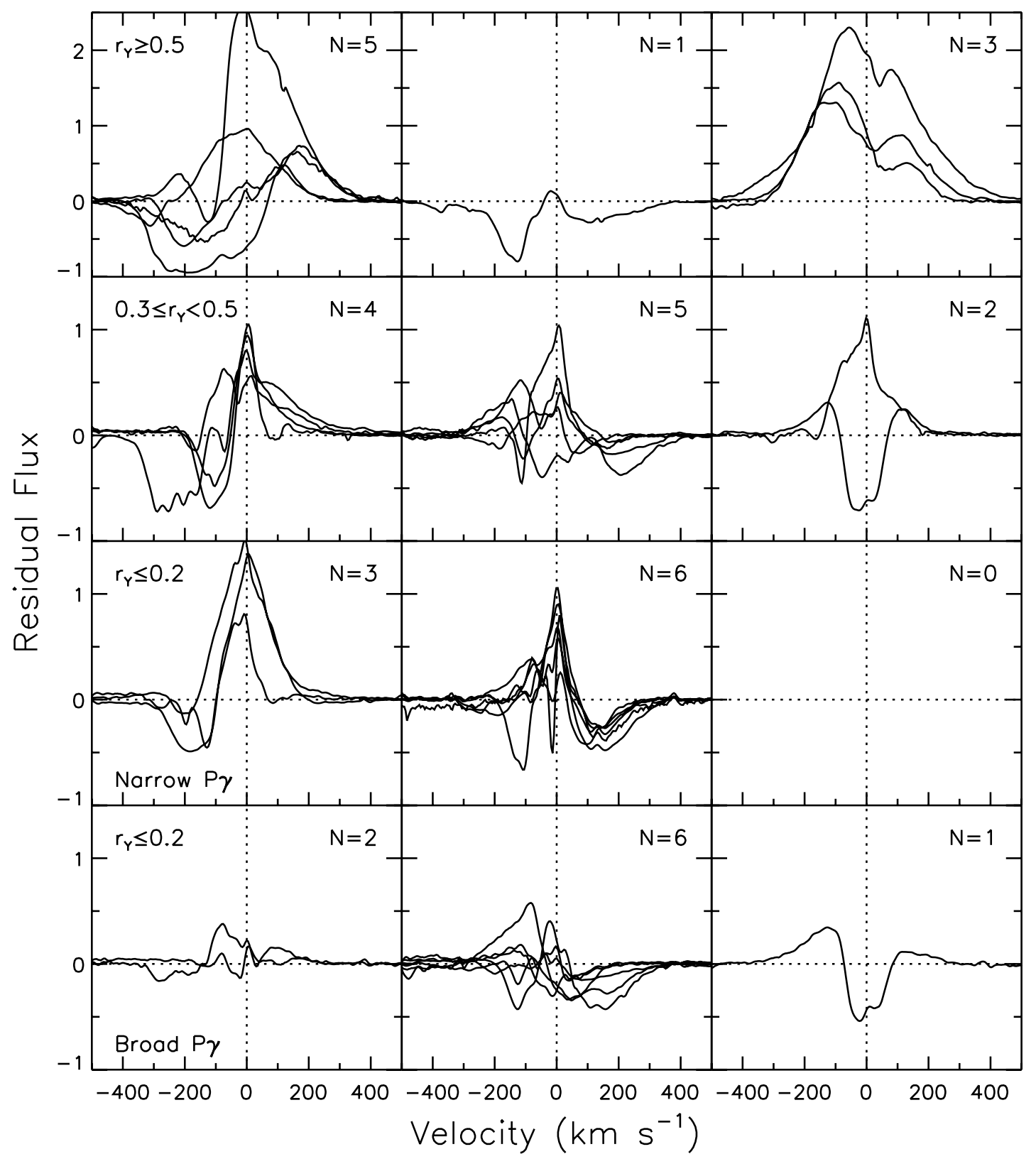

FIG. 10.-Superposed He I $\lambda 10830$ residual profiles grouped by $1 \mu \mathrm{m}$ veiling (rows) and general morphology (columns). Veiling groups are identical to Fig. 9, with the low-veiling group subdivided by the width of $\mathrm{P} \gamma$. Morphology groups are "P-Cygni-like" (left); red absorption below the continuum (middle) and other (right).

the accretion rate, whereby the narrow component from the accretion shock dominates over the broad component predominantly in stars with low accretion rates.

A comparison of $\mathrm{P} \gamma, \mathrm{He}$ I $\lambda 5876$ (from BEK01), and $\mathrm{He} \mathrm{I}$ $\lambda 10830$ profiles for the nine stars with low $1 \mu \mathrm{m}$ veiling and narrow $\mathrm{P} \gamma$ lines is shown in Figure 13. (Two were not in the BEK01 sample, but we include them in the figure for completeness.) Although the He I $\lambda 5876$ lines predate the $1 \mu \mathrm{m}$ lines by almost a decade, it is apparent that all three lines in these stars have relatively narrow emission, and for seven stars for which we have both $\mathrm{P} \gamma$ and $\mathrm{He}$ I $\lambda 5876$, the presence of a narrow core near line center is apparent in both lines. In contrast, He $\mathrm{I} \lambda 10830$ lines are considerably more complex due to the sensitivity of this transition to absorption by infalling and outflowing gas. However, in most cases the velocity extent of the blue or red absorption at He I $\lambda 10830$ appears to be matched by the broad component emission at $\mathrm{P} \gamma$ or He $\mathrm{I} 25876$.
If $\mathrm{P} \gamma$ narrow component emission, which is only seen in lowveiling stars, arises in an accretion shock then the assumption that hydrogen lines in low accretion rate stars primarily trace magnetospheric columns is suspect. A related question is where the accompanying $\mathrm{P} \gamma$ broad component is formed. In three of the lowveiling stars in Figure 13 there is an asymmetric blue wing at P $\gamma$ (AA Tau, GI Tau, and TW Hya) with a similar velocity extent to the blue absorption at He $\mathrm{I} \lambda 10830$, suggesting it may arise in the wind. A similar inference was reached by Whelan et al. (2004), who found blue wings of hydrogen lines in CTTSs to be spatially extended, requiring formation in a wind. The fact that the majority of the of CTTS P $\gamma$ profiles show a progression from broad blueshifted emission at high veiling to narrow centered emission cores with broad bases at low veiling could be explained if there is an increasing contribution from wind emission at $\mathrm{P} \gamma$ as the veiling increases. Or, if the lines are primarily formed in magnetospheric accretion columns, this behavior needs to be accounted for. In the 
TABLE 3

Blue He i 210830 Absorption in 27 CTTSs

\begin{tabular}{|c|c|c|c|c|c|}
\hline $\begin{array}{l}\text { Object } \\
\text { (1) }\end{array}$ & $\begin{array}{l}W_{\lambda} \\
(\AA) \\
(2)\end{array}$ & $\begin{array}{c}\text { Absorption } \\
(\%) \\
(3)\end{array}$ & $\begin{array}{c}\Delta V \\
\left(\mathrm{~km} \mathrm{~s}^{-1}\right) \\
(4)\end{array}$ & 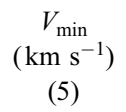 & $\begin{array}{c}V_{\max } \\
\left(\mathrm{km} \mathrm{s}^{-1}\right) \\
(6)\end{array}$ \\
\hline AS 353 A ............. & 2.8 & 60 & 220 & -80 & -300 \\
\hline CI Tau ...................... & 1.2 & 43 & 140 & -60 & -200 \\
\hline CY Tau...................... & 1.5 & 68 & 130 & -70 & -200 \\
\hline DD Tau .................. & 0.2 & 15 & $160^{\mathrm{a}}$ & -10 & -170 \\
\hline DE Tau ....................... & 0.7 & 16 & 190 & -140 & -330 \\
\hline DF Tau .................... & 1.3 & 49 & 150 & -50 & -200 \\
\hline DG Tau ................... & 1.2 & 33 & 200 & -250 & -450 \\
\hline DK Tau .................... & 3.7 & 80 & 410 & -40 & -450 \\
\hline DL Tau ................... & 0.4 & 28 & 60 & -100 & -160 \\
\hline DN Tau .................. & 0.3 & 13 & 120 & -210 & -330 \\
\hline DO Tau ................. & 2.5 & 69 & 160 & -40 & -200 \\
\hline DQ Tau .................. & 0.3 & 13 & 90 & -160 & -250 \\
\hline DR Tau ....................... & 10.7 & 95 & 470 & 70 & -400 \\
\hline DS Tau ...................... & 0.3 & 23 & 60 & -80 & -140 \\
\hline FP Таu.................... & 0.8 & 31 & 120 & $30^{\mathrm{b}}$ & -90 \\
\hline GG Tau ................ & 0.2 & 15 & 60 & -150 & -210 \\
\hline GI Tau ..................... & 0.6 & 15 & 180 & -140 & -320 \\
\hline GW Ori .................. & 4.4 & 73 & $380^{\mathrm{a}}$ & -60 & -440 \\
\hline HK Tau ................ & 0.5 & 13 & 270 & -150 & -420 \\
\hline HL Tau ..................... & 3.8 & 55 & 380 & -20 & -400 \\
\hline SU Aur..................... & 0.6 & 20 & $330^{\mathrm{a}}$ & -90 & -420 \\
\hline TW Hya .............. & 5.3 & 98 & 230 & -100 & -330 \\
\hline UY Aur.................... & 0.4 & 45 & 60 & -90 & -150 \\
\hline UZ Tau W........... & 0.3 & 10 & 190 & -60 & -250 \\
\hline V836 Tau ............ & 0.2 & 51 & 30 & 0 & -30 \\
\hline XZ Tau..................... & 1.2 & 46 & 220 & -100 & -320 \\
\hline YY Ori ..................... & 0.9 & 40 & 110 & $0^{\mathrm{b}}$ & -110 \\
\hline
\end{tabular}

Notes.-Col. (3): Percentage of continuum absorbed at the deepest point of the profile; col. (4): range of velocities for blue absorption; col. (5): minimum velocity of blue absorption; col. (6): maximum velocity of blue absorption.

${ }^{a}$ Two distinct blueshifted absorption components.

${ }^{\mathrm{b}}$ Blueshifted absorption component is blended with a redshifted absorption component; $V_{\min }$ is the local maximum in the blended region.

models it is inclination that is the major influence on line width and asymmetry, while the dominant effect of accretion rate is on line luminosity, although when combined with high temperatures, line widths will also be enhanced (Muzerolle et al. 2001a). The observed progression with veiling suggests that the paradigm of attributing hydrogen line emission to magnetospheric accretion might need to be readdressed, despite the considerable success of previous comparisons of models and observations.

There is, however, little doubt that magnetospheric accretion is present in CTTSs, attested to by the presence of redshifted IPC absorption in both hydrogen and metallic lines. Yet our data poses challenges for IPC features as well, particularly at $\mathrm{He}_{\mathrm{I}} \lambda 10830$, where the red absorption is often quite broad (several hundred kilometers per second) and deep (penetrating up to $50 \%$ of the continuum; see $\S 4$ and Figs. 4, 6, 10, and 13). This kinematic structure suggests that magnetospheric infall does not occur in a simple dipole geometry, since the funnel flow has a cross section perpendicular to the line of sight that narrows toward the star and becomes much smaller than the projected stellar surface. In the beginning of the funnel flow the speed of the gas also increases along a curved path that is more perpendicular to the line of sight than along it, so it is difficult to have the loci of points having the same line-of-sight velocity component project a large cross section for a broad velocity range, as required for the observed absorptions. Similar conclusions have also been reached by other authors investigating the geometry of $\mathrm{T}$ Tauri magnetospheres via a variety of techniques (Bouvier et al. 2006; Daou et al. 2006; Jardine et al. 2006).

We infer that although magnetospheric accretion along field lines connecting the disk and star is common in CTTSs, current models of line formation in these flows, while contributing greatly to our understanding of the star/disk interaction zone, oversimplify the actual situation. Our data suggest that the accretion shock can be a significant contributor to Paschen emission in low accretion rate objects and that a wind may contribute to the blue wings. Whether this calls into question a magnetospheric accretion origin for $\mathrm{P} \gamma$ emission in the majority of CTTSs is unclear. Although many of the observed $\mathrm{P} \gamma$ profiles have kinematic parameters in line with those predicted, the models do not account for the observed progression of $\mathrm{P} \gamma$ line widths and centroid velocities with decreasing veiling shown in Figure 9. Thus, caution is in order when deriving physical parameters of accretion flows on the sole basis of hydrogen line profiles, since it is clear that even lines of modest optical depth can have multiple sources of origin. In any case, even with multiple emission components contributing to $\mathrm{P} \gamma$ emission, each must increase in proportion to the disk accretion rate in order to reproduce the correspondence between line strength and $r_{Y}$ seen in Figure 7.

\subsection{Wind Contributions to $\mathrm{He}$ I $\lambda 10830$}

The He I $\lambda 10830$ line appears to be a promising means of studying the inner wind, given the high frequency $(71 \%)$ of CTTSs with subcontinuum blueshifted absorption. In comparison, the detection frequency of subcontinuum blueshifted absorption at $\mathrm{H} \alpha$, a traditional indicator of inner winds, is only $10 \%$ (3 of 31) among CTTSs in a sample that closely overlaps the present one (see profiles in BEK01). The most striking aspect of blueshifted absorption among CTTS He I $\lambda 10830$ profiles is their considerable diversity — in width, velocity, and penetration depth into the continuum. Blue absorption widths have breadths from as wide as $470 \mathrm{~km} \mathrm{~s}^{-1}$ to as narrow as $30 \mathrm{~km} \mathrm{~s}^{-1}$. The narrow absorption features can have blueshifts from as high as $300 \mathrm{~km} \mathrm{~s}^{-1}$ to

TABLE 4

Class I ObJects

\begin{tabular}{|c|c|c|c|c|c|c|c|}
\hline $\begin{array}{l}\text { Object } \\
\text { (1) }\end{array}$ & $\begin{array}{c}\text { Spectral Type } \\
\text { (2) }\end{array}$ & $\begin{array}{l}\text { Reference } \\
\text { (3) }\end{array}$ & $\begin{array}{l}\text { HJD } \\
(4)\end{array}$ & $\begin{array}{l}\log \dot{M}_{\text {acc }} \\
\text { (5) }\end{array}$ & $\begin{array}{l}\log \dot{M}_{\text {wind }} \\
\text { (6) }\end{array}$ & $\begin{array}{l}\text { Reference } \\
\text { (7) }\end{array}$ & $\begin{array}{c}\dot{M}_{\text {wind }} / \dot{M}_{\text {acc }} \\
\quad(8)\end{array}$ \\
\hline SVS 13 & $\mathrm{~K}:$ & 1 & $605.8,606.8$ & $-5.2^{\mathrm{a}}$ & -6.2 & 2 & $0.10^{\mathrm{a}}$ \\
\hline 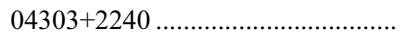 & M0.5 & 3 & 605.9 & -6.1 & -6.8 & 3 & 0.20 \\
\hline
\end{tabular}

Notes.—Col. (4): Heliocentric Julian Date (2,452,000+); col. (5): mass accretion rate in $M_{\odot} \mathrm{yr}^{-1}$; col. (6): mass-loss rate in $M_{\odot} \mathrm{yr}^{-1}$; col. (8): ratio of mass-loss rate to mass accretion rate.

${ }^{\text {a }}$ Accretion rate assumed to be 10 times the mass-loss rate.

ReFERENCES.-(1) Goodrich 1986; ; (2) Davis et al. 2001; (3) White \& Hillenbrand 2004. 

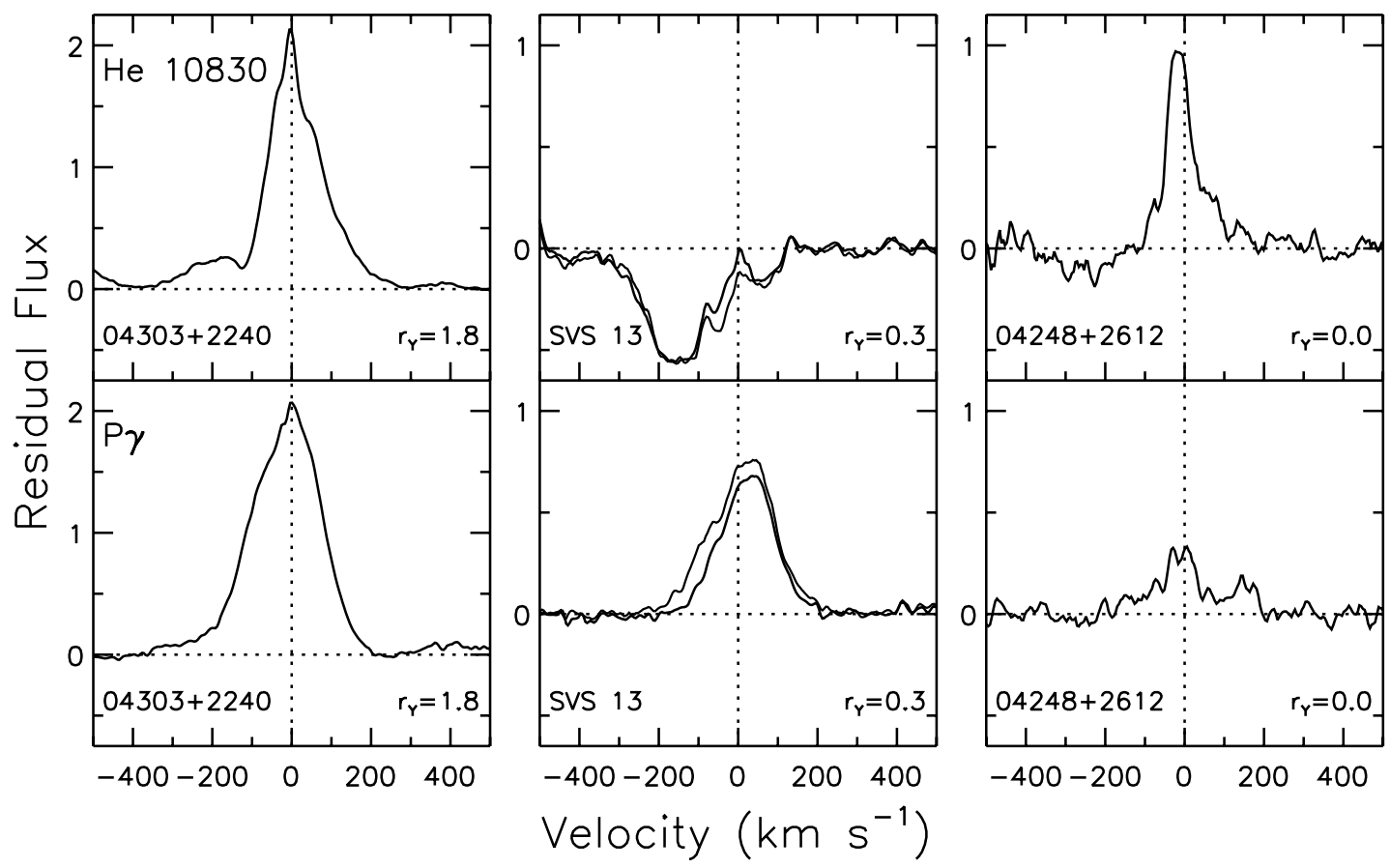

FIG. 11.-Residual He I $\lambda 10830$ and $\mathrm{P} \gamma$ profiles for the Class I sources in order of decreasing $1 \mu \mathrm{m}$ veiling $r_{Y}$. The two spectra for SVS 13 were taken a day apart; the

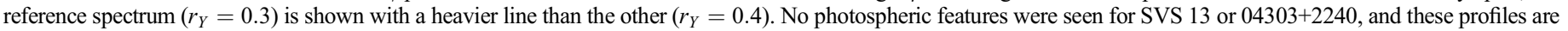
referenced relative to the velocity of ambient molecular material.

as low as tens of kilometers per second. Penetration depths can be as low as $10 \%$, but they exceed $50 \%$ in nine stars and exceed $90 \%$ in two stars (DR Tau and TW Hya). This diversity indicates that the innermost winds in accreting stars do not all have the same characteristics and that a range of wind conditions exists in these stars. However, in all cases the winds must be accretion powered, since there is no evidence for them in nonaccreting WTTSs, and the He I $\lambda 10830$ line strength, in combined emission plus absorption, is sensitive to the $1 \mu \mathrm{m}$ veiling. In this section we highlight properties of the He I $\lambda 10830$ blueshifted absorption that speak directly to the wind launch region. We are also undertaking a theoretical examination of profiles formed in winds arising both from the star and the inner disk, using Monte Carlo simulations to explore profiles formed via resonance scattering and in situ emission (J. Kwan et al. 2006, in preparation). A comparison between observed and theoretical profiles will elaborate on the points made here and demonstrate them more rigorously.

Among the stars with "P Cygni-like" profiles (Fig. 10, left column, plus SVS 13 in Fig. 11) five have blue absorption which is both very broad and very deep (SVS 13, DR Tau, AS 353A, HL Tau, and GW Ori). We argue that these absorption features require formation in a wind emerging from the star rather than the disk (see also Edwards et al. 2003). In these cases the combination of the extreme line breadth and depth reveals that all velocities in the wind, from rest to terminal velocity, intercept the $1 \mu \mathrm{m}$ continuum and scatter a significant fraction of those photons. These geometric constraints are satisfied when the source of the $1 \mu \mathrm{m}$ continuum is the stellar surface and diverging wind streamlines emerge radially away from the star, thereby absorbing continuum radiation over the full acceleration region of the wind. In contrast, for a disk wind geometry continuum photons from the star will encounter a much narrower range of velocities as they pass through the wind, which will be confined to nearly parallel streamlines emerging at an angle to the disk surface. Even in an X-wind (Shu et al. 1994), where wind streamlines open from a narrow ring in the disk, the full range of velocities in an accelerating wind would not occult the stellar surface. Central to this interpretation is the assumption that the source of the $1 \mu \mathrm{m}$ continuum photons originates from the stellar surface.

This assumption for the location of the $1 \mu \mathrm{m}$ continuum appears reasonable, as the continuum likely arises from the photosphere itself or from accretion luminosity on the stellar surface

TABLE 5

Class I Veiling and Profiles

\begin{tabular}{|c|c|c|c|c|c|c|c|c|c|c|c|c|c|}
\hline \multirow[b]{2}{*}{ Овлест } & \multirow[b]{2}{*}{$r_{Y}$} & \multicolumn{7}{|c|}{$\mathrm{P} \gamma$} & \multicolumn{5}{|c|}{ Не г $\lambda 10830$} \\
\hline & & $\begin{array}{c}V_{\text {bwing }} \\
\left(\mathrm{km} \mathrm{s}^{-1}\right)\end{array}$ & $\begin{array}{c}V_{\mathrm{BHI}} \\
\left(\mathrm{km} \mathrm{s}^{-1}\right)\end{array}$ & $\begin{array}{c}V_{c} \\
\left(\mathrm{~km} \mathrm{~s}^{-1}\right)\end{array}$ & Peak Intensity ${ }^{a}$ & $\begin{array}{c}\text { Em. W. }{ }_{\cdot \lambda} \\
(\AA)\end{array}$ & $\begin{array}{c}\text { Ab. W. }{ }_{\cdot \lambda} \\
(\AA)\end{array}$ & Ab. Type ${ }^{b}$ & $\begin{array}{c}V_{\text {bwing }} \\
\left(\mathrm{km} \mathrm{s}^{-1}\right)\end{array}$ & $\begin{array}{c}V_{\text {rwing }} \\
\left(\mathrm{km} \mathrm{s}^{-1}\right)\end{array}$ & $\begin{array}{c}\text { Em. W. } \\
(\AA)\end{array}$ & $\begin{array}{c}\mathrm{Ab} . \mathrm{W}_{\cdot \lambda} \\
(\AA)\end{array}$ & Ab. Type ${ }^{b}$ \\
\hline SVS $13 \ldots \ldots \ldots \ldots \ldots \ldots \ldots$ & 0.3 & -200 & -58 & 25 & 0.69 & 4.2 & $\ldots$ & $\ldots$ & -350 & 100 & $\ldots$ & 4.4 & $\mathrm{~b}$ \\
\hline $04248+2612 \ldots \ldots \ldots \ldots$ & 0.0 & -200 & -45 & -4 & 0.41 & 1.7 & $\ldots$ & $\ldots$ & -350 & 200 & 3.5 & 0.7 & $\mathrm{~b}$ \\
\hline $04303+2240 \ldots \ldots \ldots \ldots$ & 1.8 & -350 & -111 & -11 & 2.08 & 15.4 & $\ldots$ & $\ldots$ & -350 & 300 & 12.3 & $\ldots$ & $\ldots$ \\
\hline
\end{tabular}

\footnotetext{
a Peak emission intensity in units of the continuum.

b Classification of absorption components: (b) blue.
} 

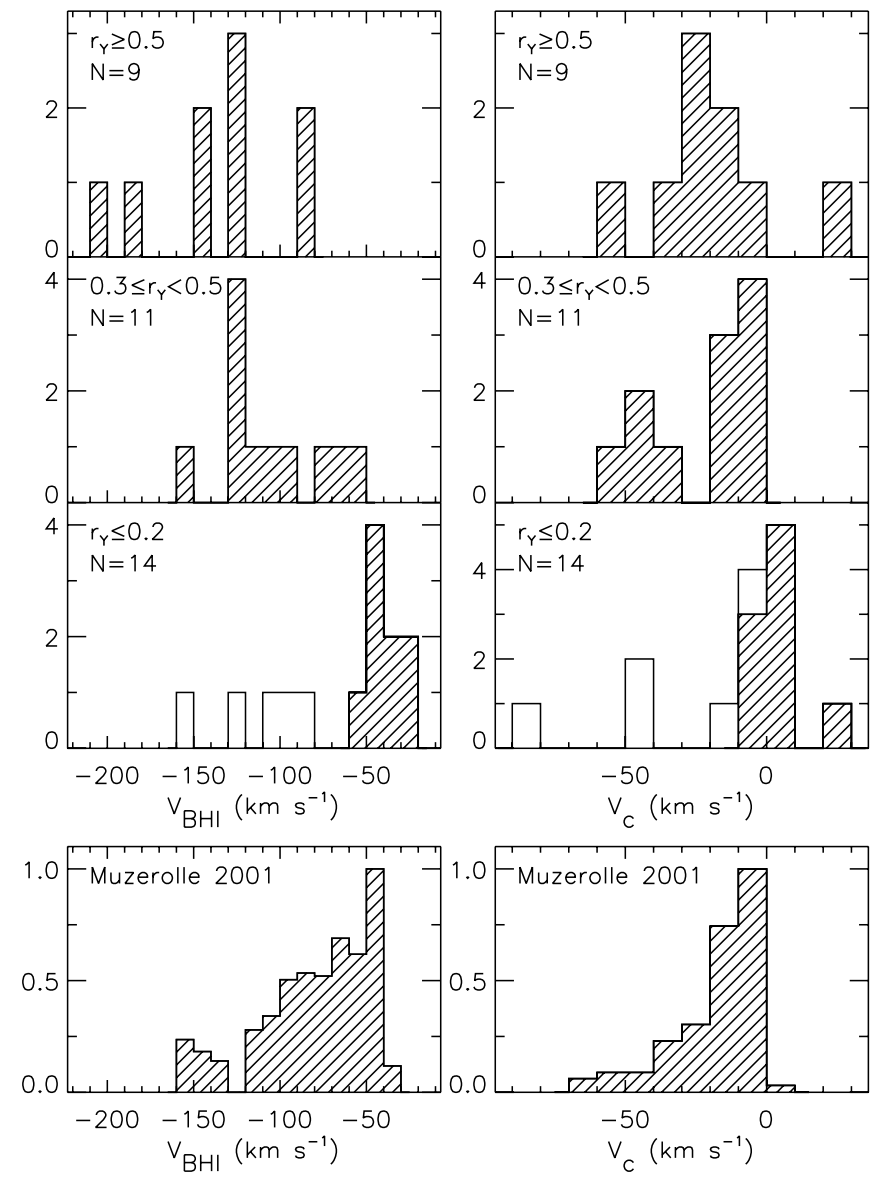

FIG. 12.-Comparison of histograms of kinematic parameters $V_{\text {BHI }}(l e f t)$ and $V_{c}$ $($ right $)$ for observed and theoretical Paschen line profiles. Observed values from $\mathrm{P} \gamma$ are separately shown in the first three rows for high, middle, and low $1 \mu \mathrm{m}$ veiling. Theoretical values from online model $\mathrm{P} \beta$ profiles (Muzerolle et al. 2001a) are in the bottom row. The distribution for the low-veiling group excludes four stars with $\mathrm{P} \gamma$ profiles that bear no resemblance to the models (BM And, SU Aur, CI Tau, and UX Tau), making comparisons meaningless. We have separately hatched the remaining five stars in the low veiling group with broad $\mathrm{P} \gamma$ profiles to emphasize the trend toward decreasing line width and centroid velocity with veiling. The model histogram is normalized to unity and weighted for a random distribution of viewing angles.

(see $\S 7$ ). Even for the largest continuum excesses we observe, $r_{Y}=2$, a third of the continuum is contributed by the photosphere. The five stars with the most broad and deep blue absorption of the $1 \mu \mathrm{m}$ continuum, and thus the strongest evidence for winds moving radially away from the star, have $r_{Y}$ values of 2 (DR Tau), 1.8 (AS 353A), 1 (HL Tau), and 0.3 (SVS 13 and GW Ori), demonstrating that similar absorption features are seen in stars with a range of continuum excesses and photospheric contributions. We refer to this wind geometry as a stellar wind, although these winds are clearly deriving their energy from processes related to disk accretion.

The above argument for stellar winds is based on the structure of the blue absorption feature in five stars. This interpretation is also consistent with their full helium profiles, which resemble line formation in a stellar wind under conditions of simple resonance scattering. Such conditions would normally result in classic P Cygni profiles with comparable emission and absorption contributions, as seen for AS353 A, HL Tau, and GW Ori. However, if the accretion disk extends close enough to the star to occult the receding flow and suppress the red emission, then a stellar wind can also account for the full profiles of DR Tau and SVS 13 , where He I $\lambda 10830$ is predominantly in absorption. This

would result if the stellar wind starts beyond the disk truncation radius, which would be favored, for example, under conditions of high disk accretion rate, resulting in smaller truncation radii (Königl 1991).

For the remaining CTTSs interpretation of the He I $\lambda 10830$ profiles is less definitive, although stellar winds are still good candidates for the profiles in the P Cygni group, such as DG Tau, DF Tau, DO Tau, and TW Hya. In these stars the blue absorption is narrow but very blueshifted and emission is the dominant contributor to the profile. Such profiles could be explained if resonance scattering plus an additional source of in situ emission is present in a stellar wind, where prominent emission would also partially fill in the absorption feature, as observed.

The possibility raised in the previous section, that the wind may contribute to broad component $\mathrm{P} \gamma$ emission, gains additional support when $\mathrm{P} \gamma$ profiles from stars with high veiling are compared with helium lines. In Figure 14 we present a figure analogous to that of Figure 13 showing $\mathrm{P} \gamma, \mathrm{He}$ I 25876 , and $\mathrm{He}_{\mathrm{I}} \lambda 10830$ profiles, but in this case for four stars with large $1 \mu \mathrm{m}$ veiling and P Cygni-like He I $\lambda 10830$ profiles. In contrast to the narrow emission seen at all three lines in the low-veiling stars, here most of the lines show broad emission (or in some cases absorption). Similarly broad, blueshifted emission is seen for both $\mathrm{P} \gamma$ and the nonsimultaneous He I 25876 lines for three of these stars (DR Tau, DG Tau, and DL Tau). In BEK01 we argued that the broad blueshifted component of $\mathrm{He}_{\mathrm{I}} \lambda 5876$ arose primarily in a wind, which Figure 14 suggests may also be contributing to $\mathrm{P} \gamma$ emission. If so, this effect may be an important contributor to the progression of $\mathrm{P} \gamma$ profile morphology described in $\S \S 3$ and 6.1. The fourth star, AS 353A, shows a more confusing relation between $\mathrm{P} \gamma$ and the nonsimultaneous He I 25876 line, where it appears as if the blue side of $\mathrm{P} \gamma$ and the red side of $\mathrm{He}$ I $\lambda 5876$ may be influenced by absorption.

In addition to the $\mathrm{P}$ Cygni profiles with blueshifted absorption likely arising from accretion powered stellar winds, other CTTS Не I $\lambda 10830$ profiles have blue absorption that is both narrow and less blueshifted, e.g., DS Tau, UY Aur, and V836 Tau. Here disk winds provide a natural explanation for the blue absorption, where stellar photons are intercepted over a narrower range of velocities as they cross parallel wind streamlines emerging from the disk. The magnitude of the blueshift will depend on the relative orientations of the disk and the line of sight, where blueshifts would be smallest for more edge-on systems. There are also a number of stars where the interpretation of the blueshifted absorption is ambiguous, and on the basis of the $\mathrm{He}$ I $\lambda 10830$ profile alone, either a stellar or disk wind might be invoked. There are also four CTTSs with no subcontinuum blue absorption and two with deep, centered absorptions, reminding us that the star-disk interface region is complex and not uniform among all accreting stars.

We consider it likely that both disk and stellar winds are present in most CTTSs, but that a combination of factors including inclination, disk geometry, accretion/outflow rates, magnetospheres, and accretion shock properties will all contribute to the formation of an observed profile. If both types of winds are present, then the stellar winds probably emerge predominantly from high latitudes. If so, the signature of stellar winds would be favored in stars seen more pole-on, while disk wind signatures would be favored in stars seen more edge-on. Unfortunately, inclination estimates for most CTTSs are not well constrained, and accretion rates are variable, so we have not tried to sort out these effects for our profiles at the present time. Although He I $\lambda 10830$ profile morphologies do not have a one-to-one correspondence with the $1 \mu \mathrm{m}$ veiling, the fact that good examples of 


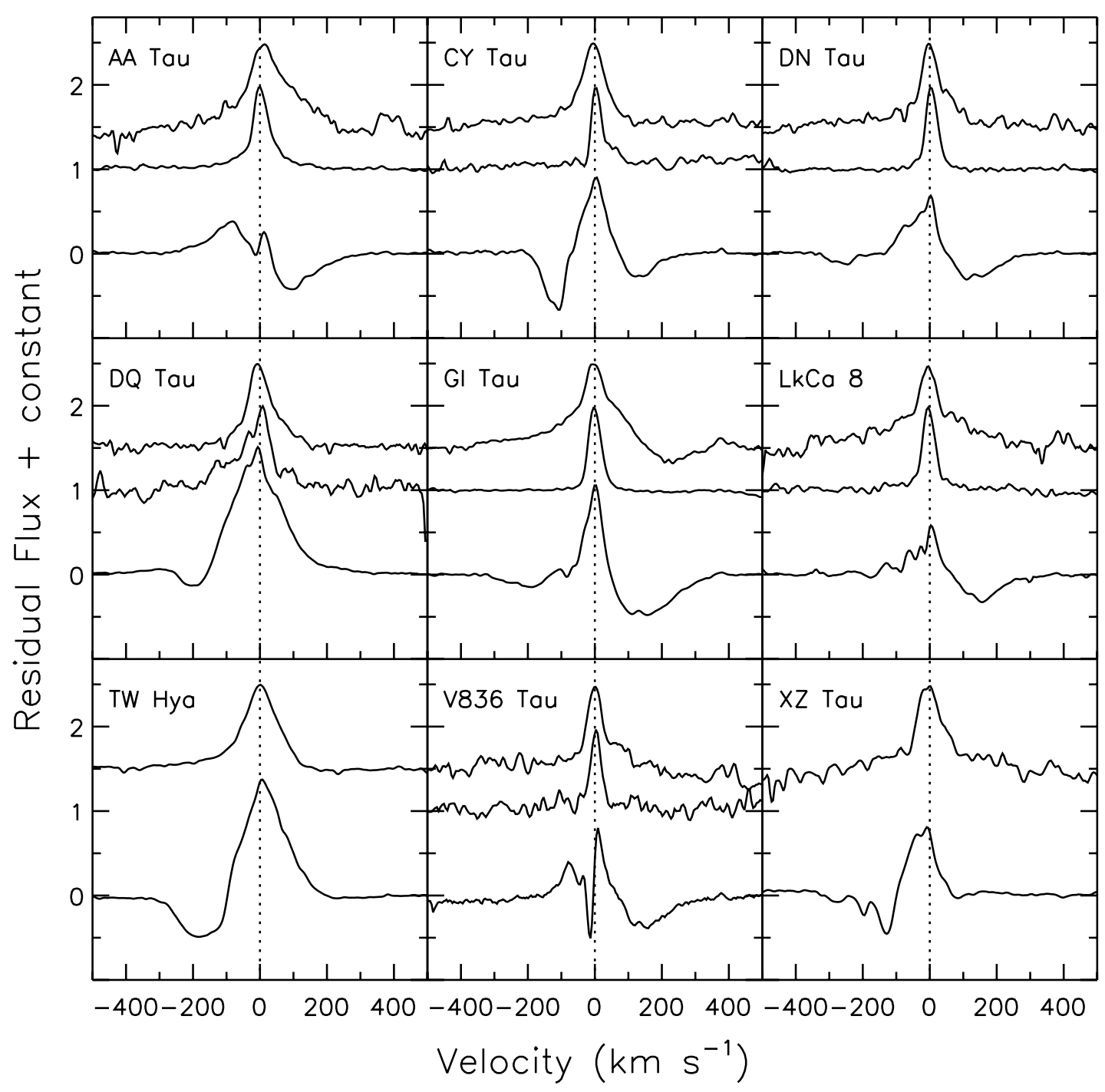

FIG. 13.-Profile comparison of $\mathrm{P} \gamma$ (top), He I 25876 (middle, from BEK01), and He I $\lambda 10830$ (bottom) for nine CTTSs with low veiling and narrow P $\gamma$ lines. P $\gamma$ and $\mathrm{He}$ I $\lambda 5876$ are normalized to their peak intensities to facilitate comparison of their kinematic structure. The He I $\lambda 10830$ profiles are in residual intensity units, except for TW Hya, which has been rescaled to half its actual peak intensity. No He I 25876 spectra are available for TW Hya or XZ Tau.

stellar wind signatures are found for stars thought to have high accretion rates and well-formed jets (e.g., SVS 13, DR Tau, AS 353A, HL Tau, and DG Tau) clarifies that the stellar wind component can be important in stars with significant accretion activity.

\section{ORIGIN OF $1 \mu \mathrm{m}$ VEILING}

Continuum emission excesses that veil photospheric features in CTTS spectra are known to come from at least two different sources. One is an optical/ultraviolet excess shortward of $0.5 \mu \mathrm{m}$ that is used to determine disk accretion rates. The spectral energy distribution of the excess emission from 0.32 to $0.52 \mu \mathrm{m}$ (Valenti et al. 1993; Gullbring et al. 1998) is very well described by theoretical models from accretion shocks formed as magnetically channeled material falls from the disk truncation radius to the stellar surface along magnetospheric field lines (Calvet \& Gullbring 1998). In the models the observed continuum emission is attributed to optically thick Paschen continua arising in the heated photosphere $(6000-8000 \mathrm{~K})$ below the shock plus optically thin emission shortward of the Balmer limit from preshock and postshock gas. There are indications that the emission excess longward of $0.5 \mu \mathrm{m}$ does not steadily decline with wavelength, as expected from the accretion shock models, but this has not been systematically studied, since it would require simultaneous observational coverage over a wide wavelength region. However, distributions of veiling among similar ensembles of CTTSs indicate that it appears to be relatively constant from 0.5 to $0.8 \mu \mathrm{m}$ (Basri \& Batalha 1990; White \& Hillenbrand 2004), suggesting that there is an additional contribution to emission excess. This leads to large uncertainties in bolometric corrections and in corresponding accretion luminosities and mass accretion rates from veilings measured in the red.

A second source of excess continuum emission recognized in accretion disk systems is found at near-infrared wavelengths from 2 to $5 \mu \mathrm{m}$ (Folha \& Emerson 1999; Johns-Krull \& Valenti 2001). This emission excess is well characterized by a single temperature blackbody with $T \sim 1400 \mathrm{~K}$ and has been successfully modeled as arising in a raised dusty rim at the dust sublimation radius in the disk (Muzerolle et al. 2003). The amount of the excess is proportional to the accretion luminosity, as the dust is heated by radiation from both the photosphere and the accretion shock. The location of the dust sublimation radius is about $0.07 \mathrm{AU}$ for a K7/MO star but can be expanded to $0.15 \mathrm{AU}$ when accretion luminosities are high (Eisner et al. 2005). 


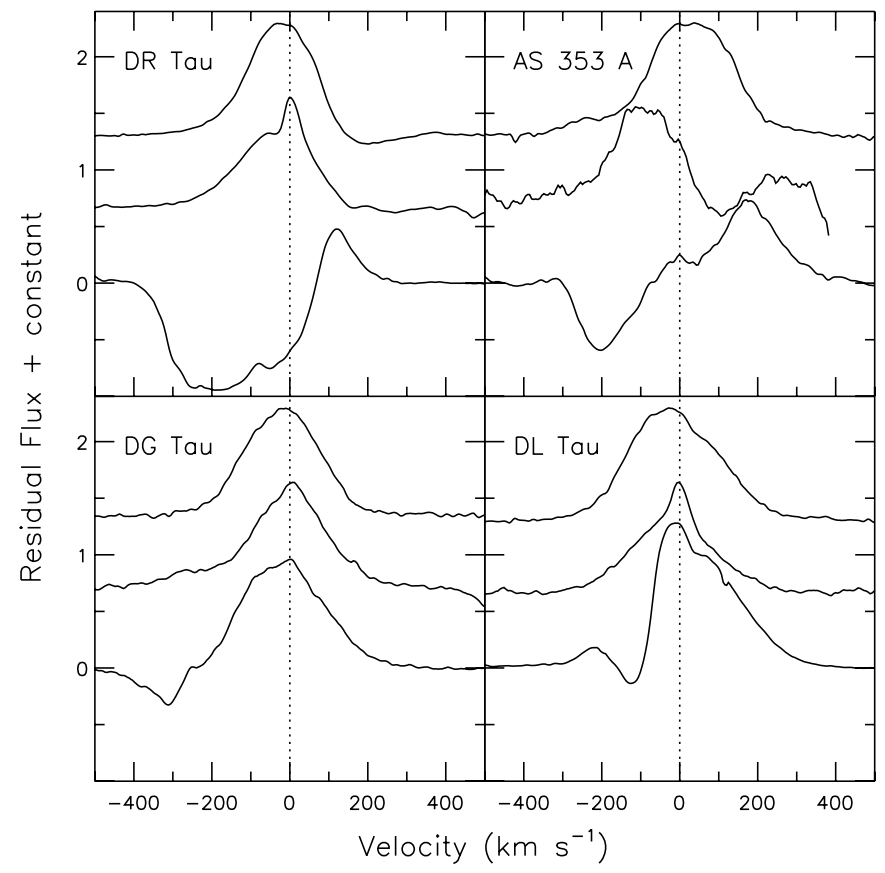

FIG. 14. - Comparison of $\mathrm{P} \gamma$ (top), $\mathrm{He} \mathrm{I} 25876$ (middle, from BEK01), and $\mathrm{He} \mathrm{I}$ $\lambda 10830$ (bottom) profiles for four CTTSs with high veiling and broad $\mathrm{P} \gamma$ lines. $\mathrm{P} \gamma$ and $\mathrm{He}$ I $\lambda 5876$ are normalized to their peak intensities to facilitate comparison of their kinematic structure. The $\mathrm{He}$ I $\lambda 10830$ profiles are in residual intensity units.

Our NIRSPEC data provide the first reconnaissance of veiling at $1 \mu \mathrm{m}$. This wavelength regime is ideally situated to provide additional insight into the behavior of continuum excess in CTTSs, since it lies between the regions where $6000-8000 \mathrm{~K}$ shocked gas and $1400 \mathrm{~K}$ warm dust are dominant contributors. However, this also corresponds to the region where the photospheric emission peaks, which will result in smaller veiling, defined as an excess relative to photospheric flux, for comparable emission fluxes. This is in fact seen, as shown in Figure 15, which compares distributions of $r_{V}, r_{Y}$, and $r_{K}$ for CTTSs, using veilings from the literature for the stars in our NIRSPEC survey. It is seen that maximum values for $r_{Y}$ are only $\sim 2$, compared to maxima of 8 for $r_{V}$ and 4 for $r_{K}$.
Similarly, $20 \%$ of stars with optical excesses do not have measurable veilings at $1 \mu \mathrm{m}$. (Veiling values and their references are compiled in Table 6). However, as we show below, the $1 \mu \mathrm{m}$ veiling on average exceeds that expected from either a 6000-8000 K accretion shock or $1400 \mathrm{~K}$ dust, and this leads us to confirm that an additional source of excess emission is present in CTTS systems.

The magnitude of the discrepancy is shown in Figure 16, which uses veiling ratios, $r_{\lambda} / r_{Y}$, to illustrate how, on average, veilings at $1 \mu \mathrm{m}$ exceed those predicted from either of the two suspected sources of continuum excess emission. The veiling ratios are plotted as a function of $T_{\text {eff }}$ in order to account for the differing proportions of photospheric contributions to the measured veiling. We also include predicted veiling ratios that would result either from $8000 \mathrm{~K}$ accretion shocks $\left(r_{B} / r_{Y}\right.$ and $\left.r_{V} / r_{Y}\right)$ or $1400 \mathrm{~K}$ warm dust $\left(r_{K} / r_{Y}\right)$. Although the CTTS ratios are compiled from nonsimultaneous veiling measurements, the ensemble of veiling ratios from many stars consistently shows $r_{Y}$ to be larger than expected from either accretion shocks or warm dust.

For example, the expected veiling ratio $r_{K} / r_{Y}$ is about 20 for warm $1400 \mathrm{~K}$ dust around a K7/M0 star. Thus, the observed maximum $r_{K}$ of 4 would only generate a $1 \mu \mathrm{m}$ veiling of $r_{Y}=$ 0.2 , considerably smaller than the observed maximum of 2 . Similarly, the observed distribution of $r_{K} / r_{Y}$ ratios indicates that on average $r_{Y}$ is an order of magnitude larger than would arise from the dust.

The corresponding situation for an $8000 \mathrm{~K}$ accretion shock predicts a veiling ratio $r_{B} / r_{Y}$ of about 7 for a $\mathrm{K} 7 / \mathrm{M} 0$ star. Thus, the maximum $r_{B}$ of 5 would correspond to an $r_{Y}$ of 0.7 , in contrast to the observed maximum of 2 . This is consistent among the group of CTTSs, in which the observed ratios again suggest $r_{Y}$ is, on average, several times larger than predicted. A similar conclusion can be drawn from the ratio $r_{V} / r_{Y}$, although the average discrepancy here is not as large. This is because by $5700 \AA$ the excess emission is already higher than expected relative to the $B$ band for an $8000 \mathrm{~K}$ accretion shock.

In summary, there is persuasive evidence suggesting that an additional source of continuum-emission excess other than 6000$8000 \mathrm{~K}$ accretion shocks and $1400 \mathrm{~K}$ warm dust is present in accreting T Tauri stars. A simple possibility is that accretion shocks are not uniform in temperature but include cooler components
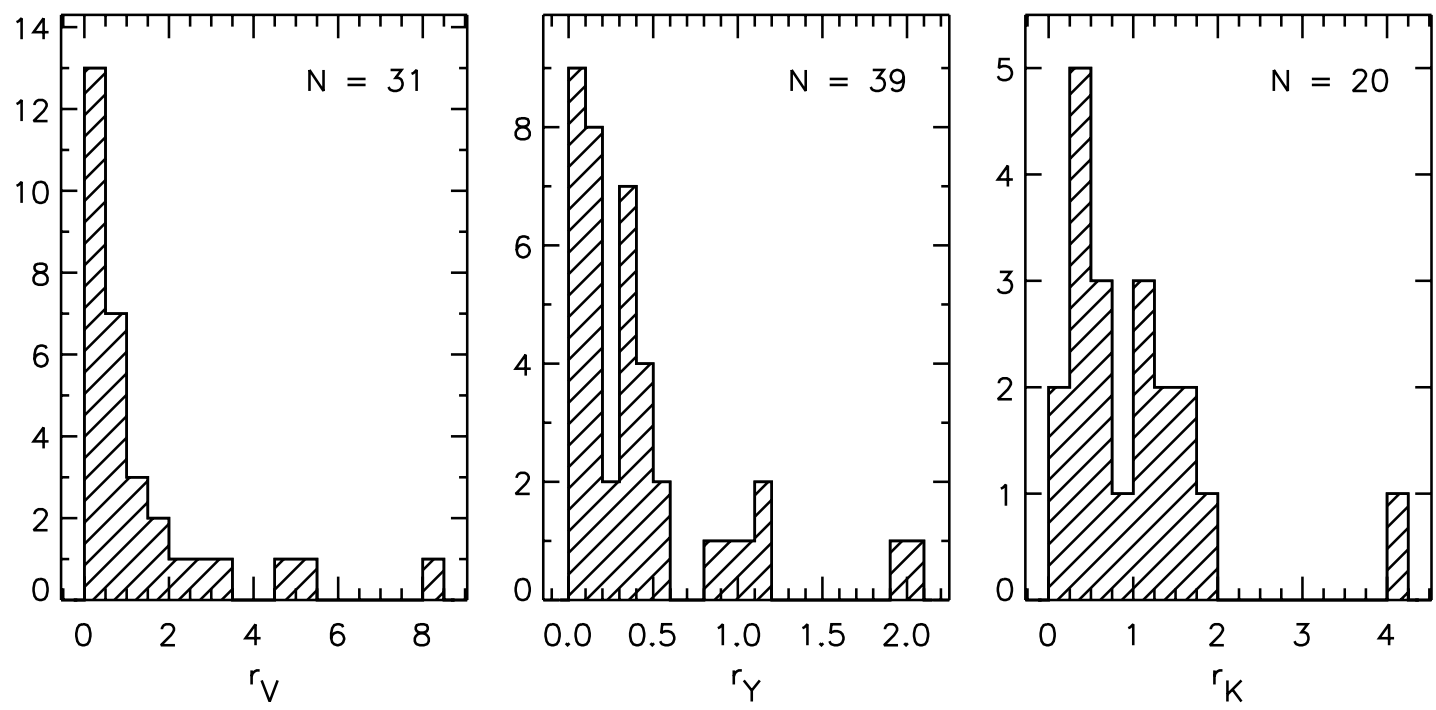

FIG. 15.-Comparison of veiling distributions at $V, Y$, and $K$. Only stars from the current survey are included, but the nonsimultaneous data for $V$ and $K$ from the literature does not include all the stars in our survey. 
TABLE 6

CTTS Mean $B, V, Y$, and $K$ Veiling

\begin{tabular}{|c|c|c|c|c|c|c|c|}
\hline Object & $r_{B}$ & $r_{V}$ & $r_{Y}$ & $r_{K}$ & $r_{B} / r_{Y}$ & $r_{V} / r_{Y}$ & $r_{K} / r_{Y}$ \\
\hline AA Tau............. & 0.27 & 0.31 & 0.15 & 0.38 & 1.80 & 2.07 & 2.53 \\
\hline AS 353 A.......... & 3.45 & 5.10 & 1.90 & $\ldots$ & 1.82 & 2.68 & $\ldots$ \\
\hline BM And ............ & $\ldots$ & $\ldots$ & 0.10 & 1.40 & $\ldots$ & $\ldots$ & 14.00 \\
\hline BP Tau .............. & 0.80 & 0.71 & 0.30 & 0.72 & 2.67 & 2.37 & 2.40 \\
\hline CI Tau ................ & $\ldots$ & 0.47 & 0.20 & $\ldots$ & $\ldots$ & 2.35 & $\ldots$ \\
\hline CW Tau............. & $\ldots$ & 1.25 & 1.15 & $\ldots$ & $\ldots$ & 1.09 & $\ldots$ \\
\hline CY Tau.............. & 0.82 & 1.20 & 0.10 & $\ldots$ & 8.20 & 12.00 & $\ldots$ \\
\hline DD Tau ............. & $\ldots$ & 2.90 & 0.10 & $\ldots$ & $\ldots$ & 29.00 & $\ldots$ \\
\hline DE Tau ............. & 0.88 & 0.57 & 0.20 & 1.05 & 4.40 & 2.85 & 5.25 \\
\hline DF Tau ............... & 1.25 & 0.93 & 0.30 & 0.81 & 4.17 & 3.10 & 2.70 \\
\hline DG Tau ............. & 2.70 & 3.15 & 0.80 & 1.43 & 3.38 & 3.94 & 1.79 \\
\hline DK Tau ............. & 0.69 & 0.49 & 0.50 & 1.62 & 1.38 & 0.98 & 3.24 \\
\hline DL Tau .............. & 2.64 & 1.64 & 1.10 & $\ldots$ & 2.40 & 1.49 & $\ldots$ \\
\hline DN Tau ............. & 0.16 & 0.07 & 0.00 & 0.21 & 6.40 & 2.80 & 8.40 \\
\hline DO Tau ............. & $\ldots$ & 4.70 & 0.30 & 1.90 & $\ldots$ & 15.67 & 6.33 \\
\hline DQ Tau ............. & $\ldots$ & 0.18 & 0.00 & 0.30 & $\ldots$ & 7.20 & 12.00 \\
\hline DR Tau .............. & 4.30 & 8.14 & 2.00 & 4.00 & 2.15 & 4.07 & 2.00 \\
\hline DS Tau ............... & 1.00 & 0.83 & 0.40 & 1.10 & 2.50 & 2.07 & 2.75 \\
\hline FP Tau.............. & $\ldots$ & 0.14 & 0.10 & 0.40 & $\ldots$ & 1.40 & 4.00 \\
\hline GG Tau ............ & 0.90 & 0.30 & 0.30 & 0.26 & 3.00 & 1.00 & 0.87 \\
\hline GI Tau ............... & 0.60 & 0.24 & 0.10 & 0.72 & 6.00 & 2.40 & 7.20 \\
\hline GK Tau ............ & 0.11 & 0.17 & 0.30 & 1.11 & 0.37 & 0.57 & 3.70 \\
\hline GM Aur............. & 0.19 & 0.22 & 0.00 & 0.30 & 7.60 & 8.80 & 12.00 \\
\hline GW Ori ............. & 0.00 & $\ldots$ & 0.30 & $\ldots$ & 0.00 & $\ldots$ & $\ldots$ \\
\hline HK Tau ............. & $\ldots$ & 1.10 & 0.40 & $\ldots$ & $\ldots$ & 2.75 & $\cdots$ \\
\hline HL Tau .............. & $\ldots$ & $\cdots$ & 1.00 & $\cdots$ & $\cdots$ & $\cdots$ & $\cdots$ \\
\hline HN Tau ............ & 0.60 & 0.76 & 0.50 & $\ldots$ & 1.20 & 1.52 & $\ldots$ \\
\hline LkCa $8 \ldots \ldots \ldots \ldots . . .$. & 0.15 & 0.15 & 0.05 & $\cdots$ & 3.00 & 3.00 & $\cdots$ \\
\hline RW Aur A ........ & 3.20 & 2.04 & 0.90 & $\ldots$ & 3.56 & 2.27 & $\cdots$ \\
\hline RW Aur B ........ & $\ldots$ & $\ldots$ & 0.10 & $\ldots$ & $\ldots$ & $\ldots$ & $\ldots$ \\
\hline SU Aur.............. & $\ldots$ & $\ldots$ & 0.00 & 0.60 & $\ldots$ & $\ldots$ & 24.00 \\
\hline TW Нyа ............ & $\ldots$ & 0.29 & 0.00 & 0.09 & $\ldots$ & 11.60 & 3.60 \\
\hline UX Tau ............. & $\ldots$ & $\ldots$ & 0.00 & $\ldots$ & $\ldots$ & $\ldots$ & $\ldots$ \\
\hline UY Aur.............. & 0.75 & 0.53 & 0.40 & 1.65 & 1.88 & 1.32 & 4.12 \\
\hline UZ Tau E .......... & $\ldots$ & 0.73 & 0.30 & $\ldots$ & $\ldots$ & 2.43 & $\cdots$ \\
\hline UZ Tau W......... & $\ldots$ & $\ldots$ & 0.10 & $\ldots$ & $\ldots$ & $\ldots$ & $\ldots$ \\
\hline V836 Tau .......... & $\ldots$ & 0.04 & 0.00 & $\ldots$ & $\ldots$ & 1.60 & $\ldots$ \\
\hline XZ Tau.............. & $\ldots$ & $\ldots$ & 0.00 & $\ldots$ & $\ldots$ & $\ldots$ & $\ldots$ \\
\hline YY Ori .............. & $\ldots$ & 1.80 & 0.40 & $\ldots$ & $\ldots$ & 4.50 & $\ldots$ \\
\hline
\end{tabular}

Notes.-Veilings are means from the following compilations: $r_{B}$ : Basri \& Batalha (1990), Gullbring et al. (1998); $r_{V}$ : Alencar \& Batalha (2002), HEG95, Muzerolle et al. (1998b); $r_{Y}$ : this paper; $r_{K}$ : Folha \& Emerson (1999), JohnsKrull \& Valenti (2001), Muzerolle et al. (2003) In calculating ratios, objects with $r_{Y}$ nondetections are set to $r_{Y}=0.025$.

than modeled to date (White \& Hillenbrand 2004). Another is radiation emerging from the region where the rotational energy of the inner disk is dissipated, which would generate lower temperatures than the accretion shocks. Determining the source of this emission and its importance in accreting systems will require simultaneous measurements over a very broad range of wavelengths.

\section{CONCLUSIONS}

Our key empirical findings from NIRSPEC $1 \mu \mathrm{m}$ spectra are as follows:

Veiling at $1 \mu \mathrm{m}$ ranges from $r_{Y}=0$ to 2 for a sample of 39 CTTSs, whose veiling at $5700 \AA\left(r_{V}=0.1-8\right)$ corresponds to 2 orders of magnitude in disk accretion rates. Approximately $20 \%$ of the CTTSs $(8 / 39)$ showed no detectable continuum excess at $1 \mu \mathrm{m}\left(r_{Y}<0.05\right)$. For the three Class I sources we ob- served, two have $1 \mu \mathrm{m}$ veiling. In the CTTSs the $1 \mu \mathrm{m}$ veiling is on average larger than expected from recognized sources of continuum excess in accreting stars, either 6000-8000 K accretion shocks on the stellar surface or $1400 \mathrm{~K}$ puffed rims at the dust sublimation radius in the disk.

The $\mathrm{P} \gamma$ line is in emission in 38/39 CTTSs with equivalent widths ranging from 0.6 to $18 \AA$ and in all three Class I sources. Profiles have central peaks, none show blueshifted absorption, and $24 \%$ of the CTTSs show redshifted absorption below the continuum. There is good correlation between $\mathrm{P} \gamma$ equivalent width and veiling, and the majority of stars also show a relation between $\mathrm{P} \gamma$ profile morphology and $r_{Y}$, with broader more blueshifted lines at high veiling. Among the 18 stars with $r_{Y} \leq 0.2$, half show $\mathrm{P} \gamma$ profiles with a two-component structure comprised of narrow centered cores $\left(\mathrm{FWHM} \sim 50 \mathrm{~km} \mathrm{~s}^{-1}\right.$ ) and a broad base $\left(\mathrm{FWHM} \sim 170 \mathrm{~km} \mathrm{~s}^{-1}\right)$.

The He I $\lambda 10830$ line, found in 38/39 CTTSs and all three Class I sources, displays a more complex profile morphology. Its resonance scattering properties make it extraordinarily sensitive to outflowing and infalling gas in the vicinity of star, with $89 \%$ of the CTTS profiles showing absorption below the continuum. Blueshifted subcontinuum absorption from a wind is found in $71 \%$ of the CTTS profiles, redshifted subcontinuum absorption from magnetospheric infall in $47 \%$, and centered subcontinuum absorption in 5\%. Blueshifted absorption is found at all veiling levels and displays considerable diversity in its velocity structure, with widths ranging from $30-470 \mathrm{~km} \mathrm{~s}^{-1}$ and depths penetrating up to $95 \%$ of the continuum. Redshifted absorption is more prevalent among stars with low veiling and when present can have widths of several hundred kilometers per second and penetrate up to $50 \%$ of the continuum.

Our key interpretive findings from NIRSPEC $1 \mu \mathrm{m}$ spectra are as follows:

We attribute the diversity of kinematic structure in He I $\lambda 10830$ subcontinuum blueshifted absorption to the presence of two genres of winds being launched from the star-disk interface region. Some CTTSs have profiles requiring a wind moving radially away from the star, while others are more readily explained as arising in winds emanating from the inner disk. There are also stars for which it is not obvious which genre of wind is dominant. Both winds must be accretion powered, since neither is seen in nonaccreting WTTSs and among the CTTSs helium strength correlates with veiling. Stellar wind signatures are found for a range of disk accretion rates, including objects of high accretion rate and well-developed jets and $\mathrm{HH}$ outflows. We consider it likely that both disk and stellar winds are present in all CTTSs, but that a combination of factors, including inclination to the line of sight, disk geometry, accretion/outflow rates, magnetospheres, and accretion shock properties, determine which genre of wind is the primary contributor to the He I $\lambda 10830$ profile in a particular star.

The high frequency of redshifted subcontinuum absorption in half of the He I $\lambda 10830$ profiles and a quarter of the $\mathrm{P} \gamma$ profiles attests to the presence of magnetospheric accretion flows. However, the breadth and depth of this feature at He I $\lambda 10830$ suggest that complex field topologies rather than simple dipole fields probably link accretion columns from the disk to the star, an idea for which there is growing observational support (Bouvier et al. 2006). Surprisingly, several aspects of $\mathrm{P} \gamma$ profile morphology suggest this line may not be solely formed in magnetospheric infall but may also have important contributions from accretion shocks and winds.

In sum, the combination of He I $\lambda 10830$ and $\mathrm{P} \gamma$ lines in the $1 \mu \mathrm{m}$ region offers rich new probes of the relation between 


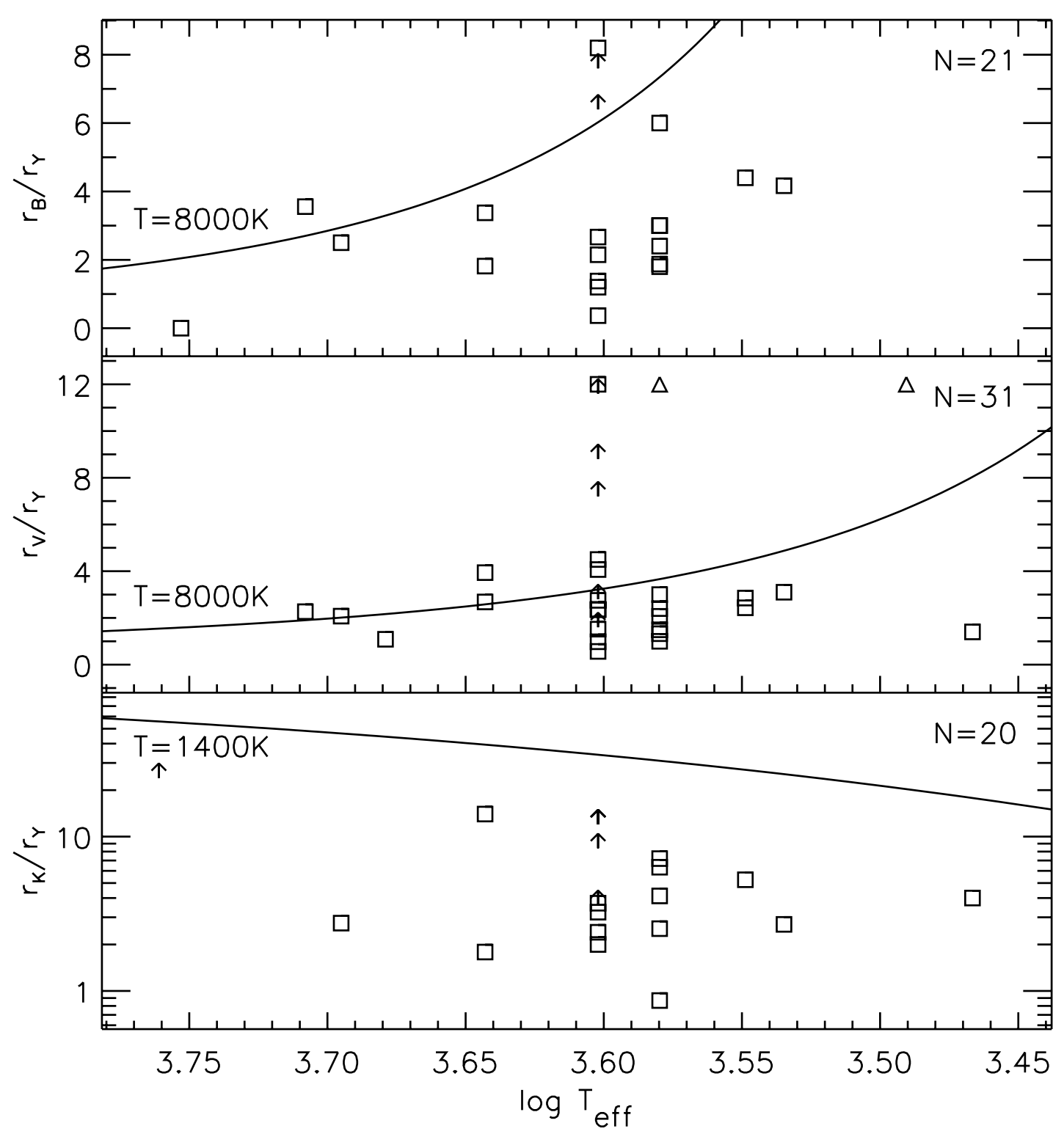

FIG. 16. - Ratios of $B$-, $V$-, and $K$-band veiling to $Y$-band veiling plotted as a function of effective temperature. Stars with measured veilings at each pair of wavelengths are plotted as open squares using mean values given in Table 6. Two stars in the $r_{V} / r_{Y}$ plot have ratios too high to fit in the figure (15.7 for DO Tau and 29.0 for DD Tau) and are shown with triangles. To include objects with nondetections at $r_{Y}$, we set $r_{Y}$ to an upper limit of $r_{Y}=0.025$. These are shown as arrows, representing lower limits on the ratio. The solid line shows the predicted ratio for a photospheric $T_{\text {eff }}$ with an excess blackbody source of $8000 \mathrm{~K}$ (top two panels) or $1400 \mathrm{~K}$ (bottom). Note that the ratios are scaled linearly in the first two panels and logarithmically in the bottom one.

accretion and outflow in young stars. This paper presents the first empirical overview of these profiles among CTTSs and demonstrates the unique power of the $\mathrm{He}$ I $\lambda 10830$ line to probe the wind launch region in accreting stars. A companion paper will present theoretical profiles for lines formed via scattering and in situ emission in winds arising from stars and from disks. The finding that at least some of the highest accretion rate CTTSs show signatures for accretion powered stellar winds clarifies that stellar winds must be an important component in accreting systems. This allows for the possibility that magnetized stellar winds rather than magnetospheric disk locking may be the means by which accreting stars spin down to rotational velocities well below break-up (Matt \& Pudritz 2005; von Rekowski \& Brandenburg 2006; Romanova et al. 2005).

Establishing mass outflow rates from $\mathrm{He}$ I $\lambda 10830$ will require good determination of physical conditions in the helium wind, which in turn will require simultaneous spectra of both $\mathrm{He}$ I $\lambda 10830$ and $\mathrm{He}_{\mathrm{I}} \lambda 5876$ to establish the conditions for helium excitation and ionization. Until these two issues have been resolved the magnitude of the contribution of winds traced by helium, either stellar or inner disk winds, to extended mass outflows and jets and to angular momentum loss from the star or disk remains uncertain.

NASA grant NAG5-12996 issued through the Office of Space Science provides support for this project. Thanks to Andrea Dupree for conversations and collaboration in an earlier phase of this work, to Russel White and Catrina Hamilton for IDL scripts, to the queue observers for the Keck-Gemini NIRSPEC program, Tom Geballe and Marianne Takamiya, for acquiring the 2001 data, and to an anonymous referee for helpful comments. 


\section{REFERENCES}

Ardila, D., Basri, G., Walter, F., Valenti, J., \& Johns-Krull, C. 2002, ApJ, 566, 1100 Alencar, S. H. P., \& Basri, G. 2000, AJ, 119, 1881

Alencar, S. H. P., Basri, G., Hartmann, L., \& Calvet, N. 2005, A\&A, 440, 595

Alencar, S. H. P., \& Batalha, C. 2002, ApJ, 571, 378

Basri, G., \& Batalha, C. 1990, ApJ, 363, 654

Batalha, C. C., Stout-Batalha, N. M., Basri, G., \& Terra, M. A. O. 1996, ApJS, 103,211

Beristain, G., Edwards, S., \& Kwan, J. 2001, ApJ, 551, 1037 (BEK01)

Bouvier, J., Alencar, S. H. P., Harries, T. J., Johns-Krull, C. M., \& Romanova, M. M. 2006, in Protostars and Planets V, ed. B. Reipurth, D. Jewitt, \& K. Keil (Tucson: Univ. Arizona Press)

Cabrit, S., Edwards, S. Strom, S. E., \& Strom, K. M. 1990, ApJ, 354, 687

Calvet, N. 1997, in IAU Symp. 182, Herbig-Haro Flows and the Birth of Low Mass Stars, ed. B. Reipurth \& C. Bertout (Dordrecht: Kluwer), 417

Calvet, N., \& Gullbring, E. 1998, ApJ, 509, 802

Calvet, N., Hartmann, L., Kenyon, S. J., \& Whitney, B. A. 1994, ApJ, 434, 330

Carr, J. S., Tokunaga, A. T., \& Najita, J. 2004, ApJ, 603, 213

Cerqueira, A. H., Velázquez, P. F., Raga, A. C., Vasconcelos, M. J., \& de Colle, F. 2006, A\&A, 448, 231

Coffey, D., Bacciotti, F., Woitas, J., Ray, T. P., \& Eislöffel, J. 2004, ApJ, 604, 758

Daou, A., Johns-Krull, C., \& Valenti, J. 2006, AJ, 131, 520

Davis, C. J., Ray, T. P., Desroches, L., \& Aspin, C. 2001, MNRAS, 326, 524

Doppmann, G. W., Jaffe, D. T., \& White, R. J. 2003, AJ, 126, 3043

Dupree, A. K., Brickhouse, N. S., Smith, G. H., \& Strader, J. 2005, ApJ, 625, L131

Edwards, S., Fischer, W., Kwan, J., Hillenbrand, L., \& Dupree, A. K. 2003, ApJ, 599, L41

Edwards, S., Hartigan, P., Ghandour, L., \& Andrulis, C. 1994, AJ, 108, 1056

Eisner, J. A., Hillenbrand, L. A., White, R. J., Akeson, R. L., \& Sargent, A. I. 2005, ApJ, 623, 952

Ferreira, J., \& Casse, F. 2004, Ap\&SS, 292, 479

Folha, D. F. M., \& Emerson, J. P. 1999, A\&A, 352, 517 2001, A\&A, 365, 90

Glassgold, A. E., Najita, J., \& Igea, J. 2004, ApJ, 615, 972

Goodrich, R. W. 1986, AJ, 92, 885

Guenther, E., \& Hessman, F. V. 1993, A\&A, 276, L25

Gullbring, E., Calvet, N., Muzerolle, J., \& Hartmann, L. 2000, ApJ, 544, 927

Gullbring, E., Hartmann, L., Briceño, C., \& Calvet, N. 1998, ApJ, 492, 323

Hartigan, P., Edwards, S., \& Ghandour, L. 1995, ApJ, 452, 736 (HEG95)

Hartigan, P., Edwards, S., \& Pierson, R. 2004, ApJ, 609, 261

Hartigan, P., Hartmann, L., Kenyon, S., Hewett, R., \& Stauffer, J. 1989, ApJS, 70,899

Hartigan, P., \& Kenyon, S. 2003, ApJ, 583, 334

Hartmann, L., Hewett, R., \& Calvet, N. 1994, ApJ, 426, 669

Herbig, G. H., \& Bell, K. R. 1988, Third Catalog of Emission-Line Stars of the Orion Population (Lick Obs. Bull. 1111; Santa Cruz: Lick Obs.)

Herczeg, G. J., Linsky, J. L., Valenti, J. A., Johns-Krull, C. M., \& Wood, B. E. 2002, ApJ, 572, 310

Herczeg, G. J., et al. 2005, AJ, 129, 2777
Hinkle, K., Wallace, L., \& Livingston, W. C. 1995, Infrared Atlas of the Arcturus Spectrum, 0.9-5.3 Microns (San Francisco: ASP)

Hirose, S., Uchida, Y., Shibata, K., \& Matsumoto, R. 1997, PASJ, 49, 193

Hollenbach, D. J., Yorke, H. W., \& Johnstone, D. 2000, in Protostars and Planets IV, ed. V. Mannings, A. P. Boss, \& S. S. Russell (Tucson: Univ. Arizona Press), 401

Jardine, M., Cameron, A. Collier, Donati, J.-F., Gregory, S. G., \& Wood, K. 2006, MNRAS, 367, 917

Johns-Krull, C. M., \& Basri, G. 1997, ApJ, 474, 433

Johns-Krull, C. M., \& Valenti, J. A. 2001, ApJ, 561, 1060

Königl, A. 1991, ApJ, 370, L39

Königl, A., \& Pudritz, R. 2000, in Protostars and Planets IV, ed. V. Mannings,

A. P. Boss, \& S. S. Russell (Tucson: Univ. Arizona Press), 759

Kwan, J., \& Tademaru, E. 1988, ApJ, 332, L41 1995, ApJ, 454, 382

Matt, S., \& Pudritz, R. 2005, ApJ, 632, L135

McLean, I. S., et al. 1998, Proc. SPIE, 3354, 566

Mundt, R., Walter, F. M., Feigelson, E. D., Finkenzeller, U., Herbig, G. H., \& Odell, A. P. 1983, ApJ, 269, 229

Muzerolle, J., Calvet, N., Briceño, C., Hartmann, L., \& Hillenbrand, L. 2000, ApJ, 535, L47

Muzerolle, J., Calvet, N., \& Hartmann, L. 1998a, ApJ, 492, 743 2001a, ApJ, 550, 944

Muzerolle, J., Calvet, N., Hartmann, L., \& D’Alessio, P. 2003, ApJ, 597, L149

Muzerolle, J., Hartmann, L., \& Calvet, N. 1998b, AJ, 116, 455 . 1998c, AJ, 116, 2965

Muzerolle, J., Hillenbrand, L., Calvet, N., Hartmann, L., \& Briceño, C. 2001b, in ASP Conf. Ser. 244, Young Stars Near Earth: Progress and Prospects, ed. R. Jayawardhana \& T. P. Greene (San Francisco: ASP), 245

Najita, J., Carr, J. S., \& Mathieu, R. D. 2003, ApJ, 589, 931

Najita, J., Edwards, S., Basri, G., \& Carr, J. 2000, in Protostars and Planets IV, ed. V. Mannings, A. Boss, \& S. S. Russell (Tucson, Univ. Arizona Press), 457

Reid, N. 2003, MNRAS, 342, 837

Reipurth, B., \& Bally, J. 2001, ARA\&A, 39, 403

Rice, W. K. M., Wood, K., Armitage, P. J., Whitney, B. A., \& Bjorkman, J. E. 2003, MNRAS, 342, 79

Richer, J. S., Shepherd, D. S., Cabrit, S., Bachiller, R., \& Churchwell, E. 2000, in Protostars and Planets IV, ed. V. Mannings, A. Boss, \& S. S. Russell (Tucson: Univ. Arizona Press), 867

Romanova, M. M., Ustyuogva, G. V., Koldoba, A. V., \& Lovelace, R. V. E. 2005, ApJ, 635, L165

Shu, F., Najita, J., Ostriker, E., Wilkin, F., Ruden, S., \& Lizano, S. 1994, ApJ, 429,781

Takami, M., Chrysostomou, A., Bailey, J., Gledhill, T. M., Tamura, M., \& Terada, H. 2002, ApJ, 568, L53

Terebey, S., Shu, F. H., \& Cassen, P. 1984, ApJ, 286, 529

Valenti, J. A., Basri, G., \& Johns, C. M. 1993, AJ, 106, 2024

von Rekowski, B., \& Brandenburg, A. 2006, Astron. Nachr., 327, 53

Whelan, E. T., Ray, T. P., \& Davis, C. J. 2004, A\&A, 417, 247

White, R. J., \& Hillenbrand, L. A. 2004, ApJ, 616, 998 